NBER WORKING PAPER SERIES

\title{
A QUANTITATIVE MODEL OF SUDDEN STOPS AND EXTERNAL LIQUIDITY MANAGEMENT
}

\author{
Ricardo J. Caballero \\ Stavros Panageas \\ Working Paper 11293 \\ http://www.nber.org/papers/w11293
}

\author{
NATIONAL BUREAU OF ECONOMIC RESEARCH \\ 1050 Massachusetts Avenue \\ Cambridge, MA 02138 \\ April 2005
}

We are grateful to Lars Hansen and conference participants at CIRJE, Tokyo, for their comments, and to Herman Bennett, Fernando Duarte and, particularly, Jose Tessada for excellent research assistance. Caballero thanks the NSF for financial support. The views expressed herein are those of the author(s) and do not necessarily reflect the views of the National Bureau of Economic Research.

(O)2005 by Ricardo J. Caballero and Stavros Panageas. All rights reserved. Short sections of text, not to exceed two paragraphs, may be quoted without explicit permission provided that full credit, including $(\odot$ notice, is given to the source. 
A Quantitative Model of Sudden Stops and External Liquidity Management

Ricardo J. Caballero and Stavros Panageas

NBER Working Paper No. 11293

April 2005

JEL No. E2, E3, F3, F4, G0, C1

\begin{abstract}
$\underline{\text { ABSTRACT }}$
Emerging market economies, which have much of their growth ahead of them, run persistent current account deficits in order to smooth consumption intertemporally. The counterpart of these deficits is their dependence on capital inflows, which can suddenly stop. In this paper we develop and estimate a quantifiable model of sudden stops and use it to study practical mechanisms to insure emerging markets against them. We first assess the standard practice of protecting the current account through the accumulation of international reserves and conclude that, even when optimally managed, this mechanism is expensive and incomplete. External insurance, on the other hand, is hard to obtain because sudden stops often come together with distress in emerging market investors themselves (the most natural insurers). Thus, one needs to find global (non-emerging-marketspecific) assets that are correlated to sudden stops. We show an example of such an asset based on the S\&P 500's implied volatility index. If added to these countries portfolios, it would significantly enhance their sudden stop risk-management strategies. In our simulations, the median gain in terms of reserves available at the time of sudden stop is around 30 percent. Moreover, in instances where the level of non-contingent reserves is low, the median gain is close to 300 percent. We also find that as countries manage to reduce the size of the sudden stops that afflict them, they should reduce their stock of reserves and significantly increase their share of contingent reserves. The main insights of the paper extend to external liquidity and liability management more generally.

Ricardo J. Caballero

Department of Economics

MIT

Room E52-252a

Cambridge, MA 02142-1347

and NBER

caball@mit.edu

Stavros Panageas

The Wharton School
\end{abstract}




\section{Introduction}

Emerging market economies, which have much of their growth ahead of them, run persistent current account deficits in order to smooth consumption intertemporally. The funding of these deficits is a perennial source of fragility since it requires ongoing capital inflows which can suddenly stop. While in many circumstances the breakdown in capital inflows simply amplify domestic deficiencies, there is extensive evidence that in many others the main culprit is not the country itself but the international financial markets' response to shocks only vaguely related to the country's actions.

The real costs of this volatility for countries that experience open crises are dramatic and well known. Less noticed, but at least as important in terms of their pervasiveness and cumulative impact, are the large costs paid by prudent economies. These economies do not fall into open crises but are forced to incur in a variety of costly precautionary measures and build large war-chests of international reserves, a trend that has only increased in the aftermath of the capital flow crises of the late 1990s. Chile and South Korea, for example, hold close to 20 percent of GDP in reserves, which contrasts with the 4 to 5 percent held by developed open economies such as Australia or Canada. How effective are reserves in smoothing the impact of sudden stops unrelated to a country's actions? How much of them should be accumulated? How fast? Are there potentially less costly financial mechanisms to deal with capital flow volatility? Who would be the natural counterpart for these mechanisms? How are these mechanisms limited by financial and collateral constraints?

These are among the most pressing questions for policy-makers and researchers in emerging market economies and the international financial institutions. Unfortunately, while there has been significant conceptual progress over the last two decades in understanding some of the limitations of financial contracting with emerging markets, there has been much less progress in providing an integral framework to analyze these questions quantitatively. In this paper we take one step in this direction.

In a nutshell, our framework considers three type of agents: An emerging market country, specialist investors, and the world capital markets at large. The essence of an emerging market economy for the problem we wish to model has two ingredients: First, its future income is significantly higher than its current income so it would like to borrow and run persistent current account deficits. Second, it has great difficulty in pledging future income to finance these deficits. Specialists can alleviate this problem but they themselves are subject to shocks that limit their ability to commit to deliver resources. These shocks, which in our model are driven by a Poisson process, trigger a period of significantly reduced capital inflows. The beginning of this period is the sudden stop stop itself, when specialists are unable to rollover all their explicit or implicit short term commitments, but it can continue even after specialists recover as countries have to rebuild their international collateral. For simplicity, we refer to the entire episode as a sudden stop. The country would like to insulate its current account financing from these sudden stops, but it cannot do so with 
its specialists since they are constrained during these events. Resorting to the world capital markets after the sudden stop takes place does not work either, since the country has very limited credibility with nonspecialists. However, world capital markets can still be used ex-ante, as long as contracts and investments are made contingent on variables that do not require emerging markets knowledge.

One of the main obstacles in building this type of structure for quantitative analysis is that it is in principle quite complex, involving several layers of potential financiers, contractual problems, and multiple state variables. Our framework preserves some of this richness but it is at the same time manageable. We make several stylized assumptions that allow us to represent the problem in terms similar to those of managing the risk associated to an exogenous non-diversifiable "income" diffusion-jump process, and is amenable to estimation.

A key simplification is that countries' and their specialists engage in growth-swaps. These swaps eliminate debt-overhang type considerations and focus the analysis on the obtention of new, uncommitted, resources. This is probably not an overly costly simplification in terms of realism, as it isolates the most fragile source of external financing. Unlike existing liabilities, uncommitted resources cannot be forced to stay in through renegotiation. It is perhaps for this reason that in practice sudden capital flow reversals are more closely associated to the current account deficit (a flow variable) than to the stock of debt (see, e.g., Calvo, Izquierdo and Mejia (2004)).

In the first part of the paper we discuss the pure reserves-management problem. Countries hoard (noncontingent) international reserves to smooth the impact of sudden stops on the current account. Since this is more or less what countries do in practice, we use this structure to estimate and calibrate the key parameters of the model. First, we use a Bayesian-hidden-state model to estimate the sudden stop processes for a small panel of emerging market economies with open capital accounts for at least two decades. Our findings indicate that sudden stops are infrequent, but recurrent and costly events. In a typical sudden stop, external funding declines by 10 percent or more, and its main impact lasts for over six years. Note that our estimation procedure is designed to capture the impact of the sudden stop beyond any initial turmoil or short run spike in interest rates. This is important because in practice current account reversals are significantly more persistent (and hence costly) than the initial impact of the sudden stop, which is what is often measured (see, e.g., Calvo et al (2004)). ${ }^{1}$ Second, we conclude from this part that holding non-contingent international reserves, as central banks do in practice, is a costly and incomplete sudden-stop insurance mechanism even when managed optimally. Reserves require large consumption sacrifices prior to sudden stops per unit

\footnotetext{
${ }^{1}$ See Caballero and Krishnamurthy (2005) for a model where sudden stops come together with a persistent decline in the country's ability to produce financial assets, and hence to attract savings. Moreover, after the initial crash, interest rates decline below the pre-crisis level, but inflows do not recover. This characterization is consistent with our empirical findings and SS-identification procedures in this paper.
} 
of protection, especially while their stock is being built. This is costly for emerging market economies, which experience limited access to external resources even during normal times. Here again, the standard practice of measuring the cost of hoarding reserves from the spread between US-Treasuries and country debt underestimates the actual opportunity cost.

This takes us to the second part of the paper, where we expand the set of investments (or contracts) countries can make. In particular, we consider the optimal inclusion of digital options on the VIX in the country's portfolio. The VIX is an index of implied volatilities extracted from options on S\&P500 firms, that simultaneously satisfies the conditions to appeal to world capital markets and to provide a good hedge against sudden stop for emerging markets: It is a developed world variable that is highly correlated with sudden stops. We discuss implementation and run the economy through simulations with the same sample paths of sudden stops used in the case with only non-contingent reserves. Relative to the latter case, the median gain in terms of reserves available at the time of sudden stop is about 30 percent. Moreover, in instances where the level of non-contingent reserves is low (lower quintile) and hence the cost of a sudden stop is high, the median gain is close to 300 percent.

In terms of dynamics, these observations mean that a country should first build the contingent part of the portfolio aggressively, adding non-contingent reserves only gradually. The reason for this strategy is that the country is particularly concerned about the worst possible event. That is, to be found without resources at the time of a sudden stop. When the stock of reserves is small, the best chance of avoiding this worst-event is by getting a large contingent payment at the time of the sudden stop. When the stock of reserves is large, the worst-event is more likely to take place if the country overcommits to contingent contracts and these do not deliver, than by holding a large amount of non-contingent reserves.

Extrapolating these dynamic implications to the cross-section, we find that as countries improve along dimensions that reduce the size of their sudden stops, they should not only reduce their reserves accumulation but also reallocate their portfolios toward more contingent instruments.

Finally, we show that the insights developed for reserves management extend more generally to external liquidity and liability management. Non-contingent debt with world capital markets has the same deficiencies that non-contingent reserves have, and hence can be improved by adding contingencies similar to those discussed for reserves. These contingencies can be obtained by either embedding them in the debt itself or by raising the contingent component of reserves.

Our paper relates to several strands of literature. The main shock that concerns us here is a sudden stop of capital inflows. The literature on sudden stops gained new life since the Asian and Russian crises. The work of Calvo (1998) describes the basic mechanics and implications of sudden stops and Calvo and Reinheart (1999) document the pervasiveness of the phenomenon. The modelling of these sudden stops as the tightening of a Kiyotaki-Moore (1997) style collateral constraint is also present in the work of Caballero 
and Krishnamurthy (2001), Arellano and Mendoza (2002) and Broner et al. (2003), among others.

As an intermediate step in developing our substantive argument and quantifying the effects we describe, we model reserves accumulation as a buffer stock model against capital flow reversals. The view that reserves can be used to cushion the impact of external shocks exists at least since Heller (1966), was enhanced by the work on precautionary savings in macroeconomics during the 1980s and has recently returned to the fore with the large accumulation of reserves exhibited by emerging markets since the crises at the end of the 1990s (see, e.g., Lee (2004)).

Importantly, the main reason for seeking insurance and hedging in our context is not income fluctuation per-se but the potential tightening of a financial constraint. This motive parallels that highlighted by Froot et al (1993) at the level of corporations, and by Caballero and Krishnamurthy (2001) for emerging markets. While the substantive themes developed in those articles differ from ours, the basic model in our paper is in many respects a dynamic version of theirs.

Closely related to our recommendations are those in the sovereign debt literature. The optimality of contingent debt and the limitations to it imposed by financial frictions are also a feature of that literature. In particular, the work of Kletzer, Newbery and Wright (1992) and Kletzer and Wright (2000), characterize feasible financial structures consistent with different degrees of commitment by a sovereign borrower and its lenders. Our collateral constraints capture features similar to those of their richer limited enforcement framework. Our paper reinforces much of the message in that literature and provides a tractable model that can be estimated and quantified.

The interaction between precautionary savings and financial constraints is also present in the closed economy framework of Aiyagari (1994). He calibrated such a model to estimates of US microeconomic income processes and other parameters and concluded that eventually agents would save enough to relax all financial constraints. Our model has similar ingredients, in that countries tend to accumulate resources over time and in that there is a level after which sudden stops would have no consequences on consumption. However, in sharp contrast with his quantitative results, ours suggests that historically countries have not gotten close to that level of savings. The main reason is the high opportunity cost of accumulating reserves for these countries, which face two financial constraints. On one hand, countries are unable to transfer enough resources from the post- to the pre-development phase. On the other, within the pre-development phase the country experiences sudden stops. The problem equivalent to that in Aiyagari is the buffer stock built to smooth the sudden stop. The long run constraint, on the other hand, raises the opportunity cost of such buffering.

Over recent years there has been a significant rise in the volatility trades, including the VIX. The finance literature has studied the impact of such trades on the performance of hedge funds and other markets participants (see, e.g., Bondarenko (2004)). From a risk management perspective, the issues faced by these 
economic agents are similar to those faced by emerging market policymakers.

We setup the model in Section 2 while constraining the precautionary options of the country to the accumulation of non-contingent reserves. In Section 3 we estimate the sudden stop process, quantify the basic model, and assess the effectiveness of reserves as a precautionary mechanism. In section 4 we let the country purchase digital options from world capital markets. Section 5 implements the extended model using the VIX as the contingent indicator on which options are written. Section 6 extends the results by adding debt and discuss contingent liability management more broadly. Section 7 concludes and is followed by several appendices.

\section{Sudden Stops and Non-contingent Protection}

While there are many important issues that arise from decentralization in economies with poor institutional development, we leave these aside and focus on the problems between the country as a whole and international investors. We study a representative agent economy with a benevolent government that seeks to maximize the expected present value of utility from consumption:

$$
\mathrm{E}\left[\int_{t}^{\infty} U\left(C_{s}\right) e^{-r(s-t)} d s\right]
$$

with $r$ being both the discount and riskless interest rate. While it is not essential, assuming a CRRA utility of consumption also simplifies the exposition:

$$
U(C)=\frac{C^{1-\gamma}}{1-\gamma}
$$

\subsection{Emerging Market Economies and World Capital Markets}

There are two features of an emerging market economy that are important for our analysis. First, its current income is low relative to its future income (it has yet to catch up), and thus it would like to borrow and run current account deficits. Second, it has difficulty pledging future income to finance these deficits.

Let $Y_{t}$ represent the country's income in the pre-development phase, and follow the geometric Brownian motion:

$$
d Y_{t}=\mu_{Y} Y_{t} d t+\sigma_{Y} Y_{t} d B_{t}
$$

with $0 \leq \mu_{Y}<r$.

Income post-development, on the other hand, is equal to:

$$
\kappa Y_{t} \quad \kappa>1 .
$$


A transition from the pre- to the post-development phase is irreversible and takes place at a random time $\tau^{G}$. The focus of the paper is on the former phase. In order to eliminate inessential time dependency, we assume that $\tau^{G}$ is governed by a Poisson process with constant hazard $g$ and independent of all other sources of uncertainty in the model. Note that $g(\kappa-1)>0$ is the difference in the expected rate of growth of a developing and a developed economy.

The swift transition and clear demarkation ensures that a country in the pre-development phase would like to borrow against its post-development income. We split potential financiers into world capital markets at large (WCM, for short), and specialists. We focus on the former in this section and discuss specialists in the next one.

World capital markets have limited connections and understanding of emerging market economies, hence they do not accept contracts contingent on variables endogenous to these economies. For now, let them just accept plain-vanilla debt. Moreover, they have only limited willingness to invest resources in any particular emerging market economy. We shall assume that a country's debt capacity with respect to WCM is capped at some finite value $W_{t}$.

The country can also accumulate international assets. Both assets and liabilities pay a return of $r$ per unit of time. The constraint with respect to WCM applies to the country's net assets, $X_{t}$ :

$$
X_{t} \geq-W_{t}
$$

Let us define reserves, $R_{t}$, to be the difference between debt capacity with respect to WCM and actual net assets:

$$
R_{t}=W_{t}+X_{t}
$$

Note that if the country is at its debt capacity, then $X_{t}=-W_{t}$ and reserves are 0 . Similarly, if $W_{t}=0$ then reserves and net uncontingent assets coincide. By construction $R_{t} \geq 0$. For now, we shall focus our attention on the case

$$
W_{t}=0
$$

and relax this simplification in Section 6. Until then, all borrowing will be done through specialists, which will be introduced shortly in the next section.

Note that given our assumptions of a continuous sample path for the income process, a stable WCM and, most importantly, a high expected growth, the country has no incentive to accumulate reserves. We establish this as our benchmark result.

Lemma 1 Assume that $W_{t}=0$ and:

$$
\mu_{Y}-\frac{\sigma^{2}(\gamma+1)}{2}>0
$$

Then, starting from $R_{t}=0$, the optimal solution is to keep $R_{t}=0$ throughout and set $C_{t}=Y_{t}$. 
That is, as long as income growth $\left(\mu_{Y}\right)$ is sufficiently strong to outweigh the precautionary savings motive $\left(\frac{\sigma^{2}(\gamma+1)}{2}\right)$, then there are no reserves accumulation in the post-development phase. This result can only be strengthened during the pre-development phase since there the expected growth rate is $\mu_{Y}+g(\kappa-1)>\mu_{Y}$. Simply put, hoarding reserves during the pre-development phase is particularly costly for an emerging market, primarily because the country as a whole is already constrained in its ability to transfer resources from the post-development phase. This means that any accumulation of reserves in aggregate carries with it a costly reduction in current consumption.

Henceforth we shall assume that condition 1 holds. This means that any accumulation of reserves will have a source different from the ingredients described up to now. We now turn to the role of specialists and their risks.

\subsection{Specialists and Sudden Stops}

Specialists are investors that have developed some expertise and connections in the country and can engage in more information-intensive contracts. Concretely, we capture this feature by allowing countries to sign contracts with specialists that are contingent on country-specific variables. In particular, we let specialists and countries write contracts contingent on the country's transition to development. With this we capture the idea that specialists big payoffs are closely tied to the country's success. In practice, these contracts may represent equity investment, FDI, the riskiest tranches of GDP-indexed bonds, or toxic-waste more generally. Later on we also consider a richer set of contingent contracts that hedge the diffusive component of income, but this we do for technical reasons and does not change the substantive message we isolate here.

Countries' can pledge up to a share $z$ of post-development output, $\kappa Y_{s}$, for all $s \geq \tau^{G}$. In aggregate - that is after netting out the multiple type of financial contracts that individuals may sign and are not of our concern in this paper — risk neutral specialists optimally engage in "swap-like" contracts with the country. At each time $t$, the specialists commit to provide resources at a rate $F_{t}$ over the next infinitesimal time interval $d t$, in exchange for receiving a promise to a stream of payments $z \kappa Y_{t}$ forever if development $\left(\tau^{G}\right)$ arrives in the interval $d t$ and 0 otherwise. By risk neutrality this stream is valued at $z \kappa Y_{t} /\left(r-\mu_{Y}\right)$.

The maximum flow that competitive specialists are willing to commit for the next $d t$ is then: ${ }^{2}$

$$
\overline{F_{t}}=\bar{f} Y_{t}
$$

\footnotetext{
${ }^{2}$ Formally, this expression can be derived from the pricing equation:

$$
r P_{t}=-\overline{F_{t}}+g\left(z \kappa Y_{t} /\left(r-\mu_{Y}\right)-P_{t}\right)
$$

and noting that a swap at the time of its inception has value $P_{t}=0$. Note that it would be trivial to add a markup to compensate specialists for the resources spent in becoming one, but this would not alter the formulae or main results in any significant way.
} 
with

$$
\bar{f} \equiv g z \kappa \frac{Y_{t}}{r-\mu_{Y}} .
$$

Assumption 1 (Limited Smoothing) $z<\frac{\kappa-1}{\kappa} \frac{r-\mu_{Y}}{r+g-\mu_{Y}}$

This ensures that the funds provided by specialists before development are less than the unpledged income after development. Thus, during non-sudden stop times, abbreviated as " $N S S$ " and sometimes referred to as "normal" times, an emerging market economy engages in as much swaps as it can:

$$
f_{t}^{N S S}=\bar{f}
$$

in order to smooth pre- and post-development consumption.

Let us now introduce the sudden stops, which are our main concern in this paper. Specialists help completing financial contracts and markets, but they can also experience shocks. We assume that there are episodes when specialists' ability to pledge resources is significantly reduced. We neither specify the particular agency problem or capital constraints that afflict specialists during sudden stops, nor let them take precautionary measures against these. We simply state the aggregate constraint implied by these shocks and focus on the country's side of the problem:

Assumption 2 (Sudden Stops) During sudden stops, specialists (collectively) can commit at most $\underline{f_{t}}<\overline{f_{t}}$ resources to the country. Specialists transit from the normal to the sudden stop stage with hazard $\lambda$ and do the reverse with hazard $\tilde{\lambda}$, both of which are independent of the transition to development.

That is, the maximum flow of resources received from the specialists before development drops to $\underline{f_{t}} Y_{t}$ during sudden stops, with

$$
\underline{f}<\bar{f}
$$

This tightening of the aggregate financial constraint can be interpreted as a drop in the share of swaps rolled over by the specialists, which are clearly binding:

$$
f_{t}^{S S}=\underline{f}
$$

For simplicity, we shall assume that if the country transits from the sudden stop to development, it transfers the effectively pledged resources $\underline{f} \kappa z / g$ per unit time to specialists. Note that we could have assumed a transfer of $\bar{f} \kappa z / g$, or anything in between, in which case not only the shadow but also the observed interest rate would rise during sudden stops. This modification is straightforward but not a first order issue in our analysis. More importantly, in the empirical section we associate the sudden stop to a period of prolonged reduction in capital inflows; in practice this may come from a combination of an initial shock to specialists 
and a longer lasting decline in the country's collateral $z$. This is straightforward to accommodate in the model, with results analogous to attributing the entire episode to specialists' problems, which is what we do throughout. ${ }^{3}$

Letting $A_{t}$ denote the sum of income and contingent flows from specialists, we can write in a mathematically compact form:

$$
A_{t}=\left(\theta^{N S S} 1\{N S S\}+\theta^{S S} 1\{S S\}+\theta^{G \mid S S} 1\{G \mid S S\}+\theta^{G \mid N S S} 1\{G \mid N S S\}\right) Y_{t}
$$

with

$$
\begin{aligned}
\theta^{N S S} & =1+\bar{f} \\
\theta^{S S} & =1+\underline{f} \\
\theta^{G \mid N S S} & =\kappa-\bar{f}\left(r-\mu_{Y}\right) / g \\
\theta^{G \mid S S} & =\kappa-\underline{f}\left(r-\mu_{Y}\right) / g
\end{aligned}
$$

where $1\{N S S\}$ and $1\{S S\}$ indicate whether the country is in normal times ("NSS"), or in a sudden stop ("SS"). Importantly, for it is behind the external crises we wish to capture,

$$
\theta^{S S}<\theta^{N S S}
$$

Finally, $1\{G \mid S S\}, 1\{G \mid N S S\}$ indicate that the country is currently developed and the transition to development took place from SS or NSS, respectively. Note that,

$$
\theta^{N S S}<\theta^{G \mid N S S}<\theta^{G \mid S S}
$$

The first of these inequalities is important since it captures the constraint to transferring resources to the emerging market (pre-development) phase. The second one is inessential, except for implicitly stating that the former constraint binds regardless of whether the particular transition into development takes place from NSS or SS.

Taking stock, we have that net assets accumulation is now described by:

$$
d X_{t}=\left(r X_{t}-C_{t}+A_{t}\right) d t
$$

Let us conclude this section with two remarks. First, by introducing specialists we have effectively introduced short term borrowing whose availability can be reversed instantaneously in a manner that is largely unrelated to country specific state variables, such as the level of debt. This captures an important

\footnotetext{
${ }^{3}$ Note, nonetheless, that we do need that specialists are a significant part of the problem, otherwise we have to consider additional insurance contracts between the country and its specialists.
} 
aspect of reality, especially for sudden stops that stem from contagion effects (see, e.g., Calvo et al (2004)). Second, we have managed to reduce the problem to one where the setup resembles closely a precautionary savings model applied to the specific jump nature of sudden stops. Thus we can use standard and well developed intuitions to analyze most of our results.

\subsection{The Problem}

We are now ready to study the reserves accumulation problem for an economy facing temporary shocks to its specialists (sudden stops). Let us now collect the ingredients into the country's optimization problem:

$$
\begin{aligned}
V\left(X_{t}, Y_{t}\right) & =\max _{C_{s}} E\left[\int_{t}^{\tau^{G} \wedge \tau^{S S}} e^{-r(s-t)} u\left(C_{s}\right) d s+e^{-r\left(\left(\tau^{G} \wedge \tau^{S S}\right)-t\right)} \tilde{V}\left(X_{\tau}, Y_{\tau}\right)\right] \\
V^{S S}\left(X_{t}, Y_{t}\right) & =\max _{C_{s}} E\left[\int_{t}^{\tau^{N S S} \wedge \tau^{G}} e^{-r(s-t)} u\left(C_{s}\right) d s+e^{-r\left(\left(\tau^{G} \wedge \tau^{N S S}\right)-t\right)} \tilde{V}^{S S}\left(X_{\tau}, Y_{\tau}\right)\right] \\
V^{G \mid S S}\left(X_{t}, Y_{t}\right) & =\max _{C_{s}} E\left[\int_{t}^{\infty} e^{-r(s-t)} u\left(C_{s}\right) d s\right] \\
V^{G \mid N S S}\left(X_{t}, Y_{t}\right) & =\max _{C_{s}} E\left[\int_{t}^{\infty} e^{-r(s-t)} u\left(C_{s}\right) d s\right] \\
& s . t . \\
\tilde{V}\left(X_{\tau}, Y_{\tau}\right) & =V^{G \mid N S S}\left(X_{\tau}, Y_{\tau}\right) 1\left\{\tau=\tau^{G}\right\}+1\left\{\tau=\tau^{S S}\right\} V^{S S}\left(X_{\tau}, Y_{\tau}\right) \\
\tilde{V}^{S S}\left(X_{\tau}, Y_{\tau}\right) & \equiv V^{G \mid S S}\left(X_{\tau}, Y_{\tau}\right) 1\left\{\tau=\tau^{G}\right\}+V\left(X_{\tau}, Y_{\tau}\right) 1\left\{\tau=\tau^{N S S}\right\} \\
d X_{t} & =\left[r X_{t}-C_{t}+A_{t}\right] d t \\
A_{t} & =\left(\theta^{N S S} 1\{N S S\}+\theta^{S S} 1\{S S\}+\theta^{G \mid S S} 1\{G \mid S S\}+\theta^{G \mid N S S} 1\{G \mid N S S\}\right) Y_{t} \\
d Y_{t} & =\mu_{Y} Y_{t} d t+\sigma_{Y} Y_{t} d B_{t} \\
X_{t} & \geq 0 \text { for all } t \geq 0 . \\
\lim _{t \rightarrow \infty} e^{-r t} X_{t} & =0
\end{aligned}
$$

where $V, V^{S S}$ and $V^{G}$ denote the value functions in the states NSS, SS and G, respectively.

In words, in post-development (in the state $G$ ) the country faces a standard precautionary savings problem. This state is absorbing, so that there can be no further transitions to the states "SS" or "NSS". The first (and final) time that development arrives is denoted by $\tau^{G}$, which occurs with hazard $g$. Prior to development, the country can be either in a sudden stop "SS" or in normal times "NSS". Transitions from normal times to sudden stops occur with a constant hazard rate of $\lambda$, at the stochastic times $\tau^{S S}$. The reverse transitions (from SS to NSS) occur at the rate $\widetilde{\lambda}$, at stochastic times $\tau^{N S S}$. Aside from the different potential transitions, the practical implication of being in any of the three regimes is that the maximum amount of resources available differs. 
Aside from the decision of how many growth-swaps to engage with specialists, which we have already discussed and solved out of the problem, the country is faced with the decision of how much to consume $\left(C_{t}\right)$. Reserves play the role of providing the country with resources during sudden stops. However, accumulating them is costly because the country is already constrained in normal times, since it cannot borrow to the full extent against its post-development income.

While a full characterization of the solution to this problem has to wait until the quantitative section, here we gauge the nature of the solution analytically.

Let us start backwards, from the post-development phase, where the problem is a conventional incomefluctuation problem. When not leading to confusion, we use the generic notation $V^{G}$ for both $V^{G \mid S S}$ and $V^{G \mid N S S}$, since they both satisfy the Bellman equation:

$$
0=\max _{c_{t}}\left\{\frac{C_{t}^{1-\gamma}}{1-\gamma}-C_{t} V_{X}^{G}\right\}+V_{X}^{G}\left(r X_{t}+\theta^{G} Y_{t}\right)-r V^{G}++V_{Y}^{G} \mu_{Y} Y_{t}+\frac{1}{2} \sigma_{Y}^{2} Y_{t}^{2} V_{Y Y}^{G}
$$

where $\theta^{G}$ should be taken to be $\theta^{G \mid S S}$ for $V^{G \mid S S}$ while it should be taken to be $\theta^{G \mid N S S}$ for $V^{G \mid N S S}$. Defining

$$
x_{t} \equiv \frac{X_{t}}{\theta^{N S S} Y_{t}} \quad c_{t} \equiv \frac{C_{t}}{\theta^{N S S} Y_{t}}
$$

noticing that

$$
V^{G}\left(X_{t}, Y_{t}\right)=\left(\theta^{N S S} Y_{t}\right)^{1-\gamma} v^{G}\left(x_{t}\right),
$$

and plugging this expression back into (8) and simplifying ${ }^{4}$, we obtain:

$$
\begin{aligned}
0= & \max _{c_{t}}\left\{\frac{c_{t}^{1-\gamma}}{1-\gamma}-c_{t} v_{x}^{G}\right\}+\left(r x_{t}+\frac{\theta^{G}}{\theta^{N S S}}\right) v_{x}^{G}-r v^{G} \\
& +\mu_{Y}\left[(1-\gamma) v^{G}-x_{t} v_{x}^{G}\right]+\frac{1}{2} \sigma_{Y}^{2}\left(-\gamma(1-\gamma) v^{G}+2 \gamma x_{t} v_{x}^{G}+x_{t}^{2} v_{x x}^{G}\right)
\end{aligned}
$$

The first order condition for consumption (normalized by potential income) is:

$$
c_{t}=\left(v_{x}^{G}\right)^{-\frac{1}{\gamma}}
$$

An immediate implication of this first order condition is that the consumption to (potential) income ratio is a function of the reserves to (potential) income ratio only.

Following similar steps in NSS, we obtain:

$$
\begin{aligned}
0= & \max _{c_{t}}\left\{\frac{c_{t}^{1-\gamma}}{1-\gamma}-c_{t} v_{x}^{N S S}\right\}+g v^{G \mid N S S}\left(x_{t}\right) \\
& +\left(r x_{t}+1\right) v_{x}^{N S S}-(r+\lambda+g) v^{N S S}+\lambda v^{S S} \\
& +\mu_{Y}\left[(1-\gamma) v^{N S S}-x_{t} v_{x}^{N S S}\right]+\frac{1}{2} \sigma_{Y}^{2}\left(-\gamma(1-\gamma) v^{N S S}+2 \gamma x_{t} v_{x}^{N S S}+x_{t}^{2} v_{x x}^{N S S}\right)
\end{aligned}
$$

\footnotetext{
${ }^{4}$ For details see Duffie et al. (1997)
} 
Once again, the first order condition for consumption is

$$
c_{t}=\left(v_{x}^{N S S}\right)^{-\frac{1}{\gamma}}
$$

Finally, we obtain the Bellman equations for the SS region:

$$
\begin{aligned}
0= & \max _{c_{t}}\left\{\frac{c_{t}^{1-\gamma}}{1-\gamma}-c_{t} v_{x}^{S S}\right\}+g v^{G \mid S S}\left(x_{t}\right) \\
& +\left(r x_{t}+\frac{\theta^{S S}}{\theta^{N S S}}\right) v_{x}^{S S}-(r+\widetilde{\lambda}+g) v^{S S}+\widetilde{\lambda} v^{N S S} \\
& +\mu_{Y}\left[(1-\gamma) v^{S S}-x_{t} v_{x}^{S S}\right]+\frac{1}{2} \sigma_{Y}^{2}\left(-\gamma(1-\gamma) v^{S S}+2 \gamma x_{t} v_{x}^{S S}+x_{t}^{2} v_{x x}^{S S}\right)
\end{aligned}
$$

which has a first order condition similar to that in the NSS region:

$$
c_{t}=\left(v_{x}^{S S}\right)^{-\frac{1}{\gamma}} .
$$

It is instructive to study and compare the first order conditions across the regions, in the (positive) neighborhood of $x_{t}=0$. It follows from the condition $\theta^{G \mid S S}>\theta^{G \mid N S S}>\theta^{N S S}>\theta^{S S}$ that:

$$
v_{x}^{G \mid S S}(0)<v_{x}^{G \mid N S S}(0)<v_{x}^{N S S}(0)<v_{x}^{S S}(0)
$$

The last inequality implies that consumption drops at the sudden stop, while the next to last implies that the country is not smoothing pre- and post-development consumption. It turns out that, as we show in the next section, these features of the solution carry over to most of the empirically relevant range of reserves.

\section{Quantitative Analysis}

In this section we estimate the main parameters of the model, calibrate others, and assess the optimal reserves strategy quantitatively.

\subsection{The Sudden Stop Process}

The core of our analysis is the sudden stop process. In this section we estimate the main parameters of such process: $\lambda, \tilde{\lambda}$, and $\theta^{S S} / \theta^{N S S}$ using data from 1983 to 2003 for six representative emerging market economies (see the Appendix for selection criterion): Chile, Colombia, Indonesia, Malaysia, Mexico, and Thailand.

The first step is to find empirical counterparts for the processes describing available resources during NSS and SS. For this, we note that in the model these resources can be decomposed into income, $Y_{t}$, and financial flows, $\left(\theta_{t}-1\right) Y_{t}$. In practice, there are several additional complexities in doing such a decomposition. These stem from the existence of multiple goods whose relative prices change during the transitions between NSS 
and SS and viceversa, the presence of temporary terms of trade shocks, and endogenous domestic output declines during sudden stops. The Data Appendix describes our methodology to deal with these issues. In a nutshell, we approximate $Y_{t}$ with the permanent component of domestic national income, and $\left(\theta_{t}-1\right) Y_{t}$ with the sum of capital flows in terms of imported goods and the transitory component of exports and terms of trade effects. Our main left hand side variable is the ratio of these two, which can be loosely interpreted as external financing over "normal" pre-development income:

$$
\psi_{i t} \equiv \theta_{i t}-1=\frac{\left(\theta_{i t}-1\right) Y_{i t}}{Y_{i t}} .
$$

In our model, $\psi$ can take only two values: $\left(\theta^{N S S}-1\right)$ and $\left(\theta^{S S}-1\right)$. Since this this is too stark a characterization for actual data, we assume that that $\psi$ is observed with (state contingent) noise,

$$
\widetilde{\psi}_{i t}=\psi_{i}\left(s_{i t}\right)+e_{i t}\left(s_{i t}\right)
$$

with

$$
e_{i t}\left(s_{i t}\right) \sim N\left(0, \sigma_{e}^{2}\left(s_{i t}\right)\right), \quad s_{i t} \in\{S S, N S S\} .
$$

Then $\widetilde{\psi}_{i t}$ follows a process described by a standard regime-switching model a la Hamilton $(1989,1990)$, with parameters: $\left\{\psi_{i}^{N S S}, \psi_{i}^{S S}, \sigma_{e, i}^{N S S}, \sigma_{e, i}^{S S}, p_{i}(N S S \rightarrow S S), p_{i}(S S \rightarrow N S S)\right\}$, such that:

$$
\begin{aligned}
p_{i}(N S S & \rightarrow S S)=\operatorname{Pr}\left(s_{i, t+\Delta}=S S \mid s_{i, t}=N S S\right)=1-e^{-\lambda_{i} \Delta} \\
p_{i}(S S & \rightarrow \quad N S S)=\operatorname{Pr}\left(s_{i, t+\Delta}=N S S \mid s_{i, t}=S S\right)=1-e^{-\widetilde{\lambda}_{i} \Delta}
\end{aligned}
$$

where we have approximated the continuous time model by its discrete analog assuming that there can be at most one transition in a time interval of $\Delta$.

For the calibration exercises we convert annual transition probabilities into annual frequencies by setting:

$$
\begin{aligned}
& \lambda=-\log [1-P(N S S \rightarrow S S)] \\
& \tilde{\lambda}=-\log [1-P(S S \rightarrow N S S)]
\end{aligned}
$$

Given the limited number of SS observations we have for each country and the highly nonlinear nature of the hidden states model we are estimating, we use a Bayesian approach based on a Gibbs Sampler (see Kim and Nelson 1999). We describe the precise procedure in the Appendix, but the main steps are as follows:

- We fix arbitrary starting parameters.

- Conditional on these parameters, we filter the data using Hamilton's algorithm to find the posterior probability that at a given point in time each country is in a SS or in NSS.

- We draw a sample path from this joint distribution. Such a path takes the value 1 if a given country is in a SS and 0 otherwise. 


\begin{tabular}{cccccccc}
\hline \hline & Average & Std Dev & $5 \%$ & $25 \%$ & $50 \%$ & $75 \%$ & $95 \%$ \\
\hline$\lambda$ & 0.19 & 0.050141 & 0.11337 & 0.15434 & 0.1868 & 0.22153 & 0.27772 \\
$\widetilde{\lambda}$ & 0.14769 & 0.046718 & 0.079083 & 0.11403 & 0.14322 & 0.17685 & 0.23111 \\
\hline \hline
\end{tabular}

Table 1: Pooled Estimates using 1983-2003: Poisson Parameters.

- We condition on these paths (one for each country) and derive the joint posterior distribution of the parameters. We require only that parameters related to $p_{i}(N S S \rightarrow S S), p_{i}(S S \rightarrow N S S)$ are the same across all countries, while the rest of the parameters can vary across countries. These parameters are key for the quantitative exercises that follow, while they seem to be the hardest ones to measure based on time series observations alone. Hence we exploit the cross sectional dimension of the panel in order to increase the precision of these estimates. Throughout we use uninformative priors.

- Finally, we draw a new set of parameters from this posterior distribution and iterate the procedure several thousand times. By standard results in Bayesian computation, the stationary distribution of the sampled parameters coincides in law with the posterior distribution of the parameters.

Tables 1 and 2 present the estimates we obtain form this procedure for the parameters $\psi_{i}^{N S S}, \eta_{i} \equiv \psi_{i}^{S S}-$ $\psi_{i}^{N S S}, \lambda_{i}, \widetilde{\lambda}_{i} \cdot{ }^{5}$ Note that from the first two parameters we can recover $\frac{\theta_{i}^{S S}}{\theta_{i}^{N S S}}$ since:

$$
\frac{\theta_{i}^{S S}}{\theta_{i}^{N S S}}=1+\frac{\eta_{i}}{1+\psi_{i}^{N S S}}
$$

Based on the medians, we conclude that sudden stops are large, leading to declines in available resources typically beyond 10 percent, last for about 6.5 years years and occur about every 12 years (about 5.5 years after exiting the previous sudden stop). As we said in the introduction, our measure of sudden stop is meant to capture not only the initial spike in interest rates and turmoil but also the effects of sudden stops that remain even after the main event. Our estimates suggest that these effects can be quite large: Countries take a long time in resuming significant borrowing after experiencing a severe capital flow reversals.

Finally, Figure 2 summarizes the output of the corresponding Gibbs sampler run for our economies. It illustrates the path of the share of economies with posterior probabilities of being in sudden stop above 0.5 during a given period. It is apparent from this figure that our procedure does capture the events one would want it to capture when studying sudden stops.

\footnotetext{
${ }^{5}$ We also estimated for a sub-sample from the 1990s using quarterly data for a broader set of countries. The results were very similar except for $\widetilde{\lambda}$, which were cut in half. However, this estimator is too affected by end of sample condition in a short sample (if we trim the last two years, the estimates become very similar to those of the 1983-2003 sample that we report).
} 


\begin{tabular}{lccccccc}
\hline \hline Chile & Average & Std Dev & $5 \%$ & $25 \%$ & $50 \%$ & $75 \%$ & $95 \%$ \\
\hline$\psi^{N S S}$ & 0.066 & 0.024 & 0.031 & 0.049 & 0.063 & 0.081 & 0.108 \\
$\eta$ & -0.091 & 0.030 & -0.132 & -0.109 & -0.094 & -0.076 & -0.041 \\
\hline Colombia & & & & & & & \\
\hline$\psi^{\text {NSS }}$ & 0.059 & 0.008 & 0.044 & 0.055 & 0.060 & 0.065 & 0.070 \\
$\eta$ & -0.051 & 0.009 & -0.064 & -0.057 & -0.052 & -0.047 & -0.036 \\
\hline Mexico & & & & & & \\
\hline$\psi^{N S S}$ & 0.078 & 0.010 & 0.061 & 0.072 & 0.079 & 0.085 & 0.094 \\
$\eta$ & -0.084 & 0.015 & -0.106 & -0.093 & -0.085 & -0.075 & -0.059 \\
\hline Indonesia & & & & & & \\
\hline$\psi^{N S S}$ & 0.043 & 0.006 & 0.033 & 0.039 & 0.043 & 0.047 & 0.053 \\
$\eta$ & -0.066 & 0.009 & -0.081 & -0.072 & -0.067 & -0.061 & -0.049 \\
\hline Malaysia & & & & & & \\
\hline$\psi^{N S S}$ & 0.114 & 0.020 & 0.081 & 0.101 & 0.114 & 0.127 & 0.146 \\
$\eta$ & -0.164 & 0.023 & -0.199 & -0.178 & -0.164 & -0.149 & -0.126 \\
\hline Thailand & & & & & & \\
\hline$\psi^{N S S}$ & 0.098 & 0.019 & 0.064 & 0.085 & 0.099 & 0.111 & 0.127 \\
$\eta$ & -0.146 & 0.023 & -0.179 & -0.161 & -0.147 & -0.133 & -0.107 \\
\hline \hline
\end{tabular}

Table 2: Country Specific Parameters 1983-2003: Normal Level of Resources and Sudden Stops. 

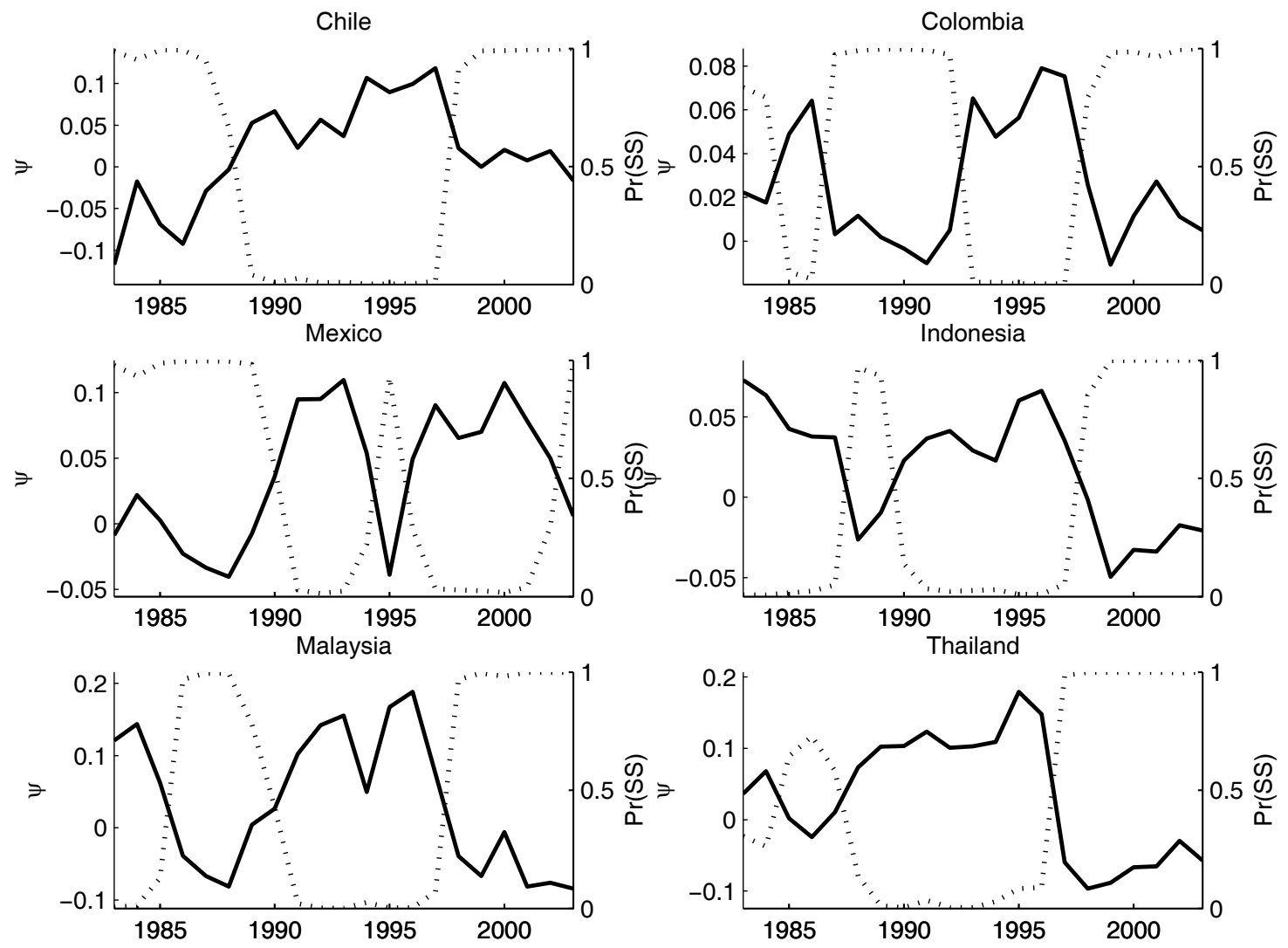

Figure 1: $\psi^{i}$ and posterior probability of being in a SS for 6 different countries. 


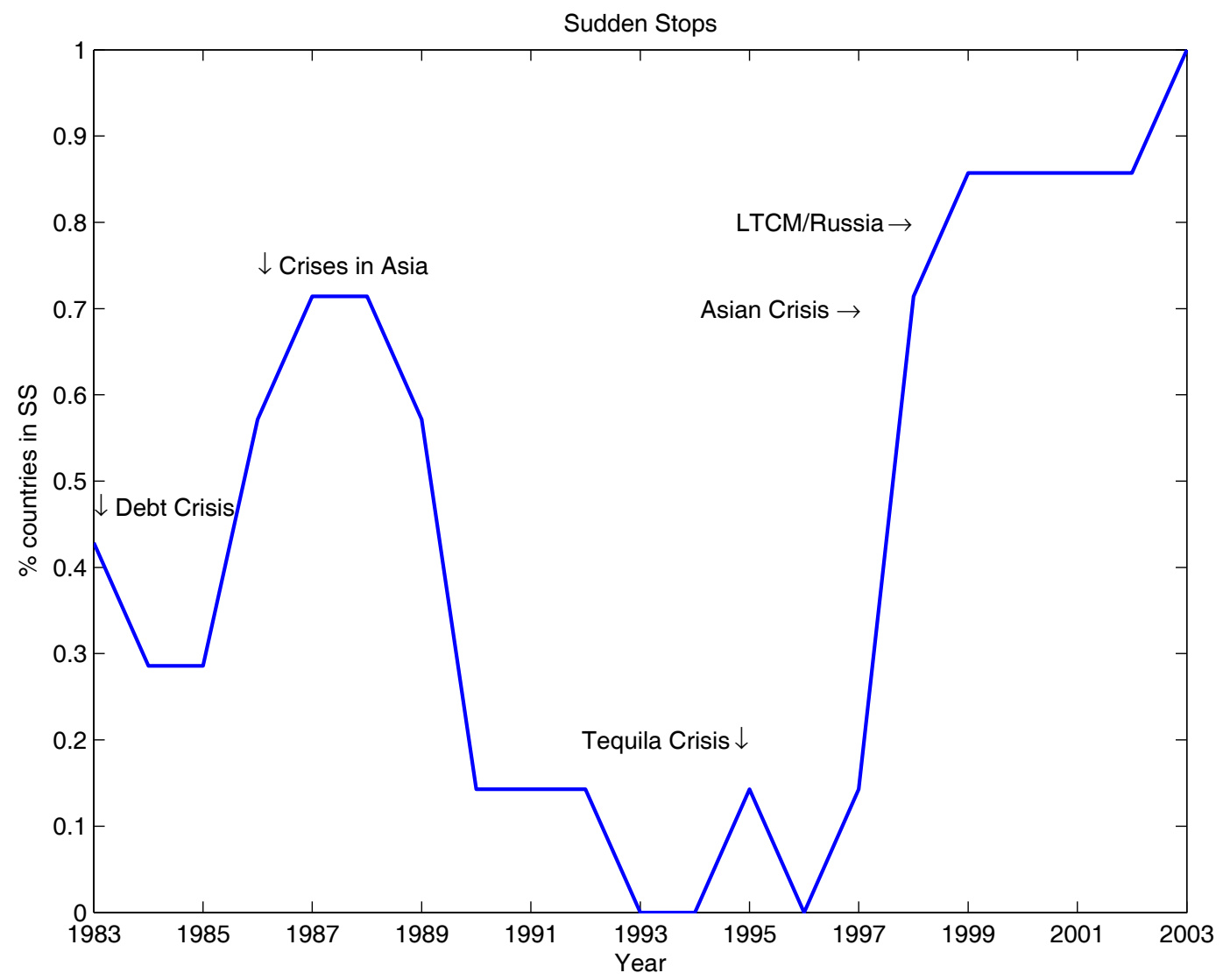

Figure 2: Fraction of Economies in SS 


\subsection{Other parameters}

In addition to $\lambda, \tilde{\lambda}$ and $\theta^{N S S} / \theta^{S S}$ we need to determine $r, \mu_{Y}, \sigma_{Y}, g, \alpha, \gamma, \theta^{G \mid S S} / \theta^{S S}$ and $\theta^{G \mid N S S} / \theta^{N S S}$ before simulating the model. Within reasonable ranges, the choice of $r$, which represents both the interest and the discount rate, is not of first order importance; we simply set it to 0.04. The post-development growth rate $\mu_{Y}$ is set to 0.018 , which is approximately the rate of growth of per-capita consumption in the US over the very long sample considered in Campbell and Cochrane (1999). We set $\sigma_{Y}=0.05$, which is higher than the developed economies volatility in national income because emerging markets also have significant terms of trade fluctuations. The parameter $g$ is set to 0.025 , which matches the speed of convergence estimated by Barro and Sala-i-Martin (2003). We set $\theta^{G \mid N S S} / \theta^{N S S}$ to 2 , so that the expected rate of growth of income in an emerging market economy during normal times is:

$$
\mu_{Y}+g \log \left(\frac{\theta^{G \mid N S S}}{\theta^{N S S}}\right)=3.7 \%
$$

which is on the conservative end of an emerging market economy's expected growth during normal times. The parameter $\theta^{G \mid S S} / \theta^{S S}$ follows from the previous ones since:

$$
\frac{\theta^{G \mid S S}}{\theta^{S S}}=\frac{r-\mu_{Y}}{g}\left(\frac{\theta^{N S S}}{\theta^{S S}}-1\right)+\frac{\theta^{G \mid N S S}}{\theta^{N S S}} \frac{\theta^{N S S}}{\theta^{S S}}
$$

We used $\gamma$ to match a reasonable stationary level of central bank reserves. Consider the dynamics of

$$
\begin{aligned}
d \log \left(x_{t}\right) & =d \log \left(\frac{X_{t}}{\theta^{N S S} Y_{t}}\right)=\left(r-\left(\mu_{Y}-\frac{\sigma^{2}}{2}\right)-\frac{C_{t}}{X_{t}}+\frac{\theta^{N S S} Y_{t}}{X_{t}}\right) d t-\sigma d B_{t}= \\
& =\left(r-\left(\mu_{Y}-\frac{\sigma^{2}}{2}\right)-\frac{C_{t}}{\theta^{N S S} Y_{t}}\left(\frac{\theta^{N S S} Y_{t}}{X_{t}}\right)+\frac{\theta^{N S S} Y_{t}}{X_{t}}\right) d t-\sigma d B_{t}= \\
& =\frac{1}{x_{t}}\left(r-\left(\mu_{Y}-\frac{\sigma^{2}}{2}\right) x_{t}-c_{t}+1\right) d t-\sigma d B_{t}
\end{aligned}
$$

Let us define the "steady state" level of $x$ as:

$$
x^{*}=\min _{x}\left\{\left(r-\left(\mu_{Y}-\frac{\sigma^{2}}{2}\right)\right) x-c_{t}+1=0\right\}
$$

Beyond this level, the process for $x_{t}$ has a negative drift and reserves start to decline on average. By setting $\gamma=8$, we obtain an $x^{*}=0.2$, which is a reasonable estimate for the maximum desired level of reserves for countries not attempting to use reserves accumulation with goals other than smoothing sudden stops (such as maintaining an undervalued currency, and so on).

Finally, we use $\eta=-0.1, \psi^{N S S}=0.08$ as a base case scenario in this section. These correspond roughly to the median values of these parameters across the countries that we consider. The values for $\lambda, \tilde{\lambda}$ are taken from the estimations of the previous section. Table 3 summarizes the values of the parameters used. 


\begin{tabular}{lccc}
\hline \hline$\gamma$ & 8 & $\lambda$ & 0.211 \\
$\delta$ & 0.04 & $\tilde{\lambda}$ & 0.158 \\
$r$ & 0.04 & $\eta$ & -0.1 \\
$\mu$ & 0.018 & $g$ & 0.025 \\
$\sigma$ & 0.05 & $\psi$ & 0.08 \\
\hline \hline
\end{tabular}

Table 3: Parameters used in Simulations.

\subsection{Implications}

Figure 3 depicts the policy functions. The upper curve represents consumption during NSS and the lower one represents consumption during SS, both as a function of the level of reserves. The vertical distance between these two curves illustrates the instantaneous drop in consumption once the country transits into $S S$. In the neighborhood of 0 this drop is largest and around $7.5 \%$, and it declines as the level of reserves rises. Because the drop is very large when the country has no reserves, precautionary savings is also maximum a this point, which is reflected in the difference between one and $c^{N S S}$.

Note that the policy functions $c^{N S S}(x)$ and $c^{S S}(x)$ do not converge. This is because, as we discussed in the calibration section, once the country reaches $x^{*}=0.2$, it stops accumulating reserves. If reserves exceed this level, the country rather consume them than use them to further smooth sudden stops. The opportunity costs of reserves is simply to great to purse full insurance. Despite the large costs associated to sudden stops, the strength of the other financial constraint (the inability to pledge a significant share of future, and higher, income) raises the opportunity cost of storing reserves enough to make it (constrained) optimal to remain somewhat unprotected against these sudden stops.

Panels (a) and (b) in Figure 4, plot the paths of consumption and reserves, respectively, for a case where the country starts with 0 reserves, then a sudden stop takes place exactly at its expected time, $1 / \lambda$, and the sudden stop lasts exactly its expected time, $1 / \widetilde{\lambda}$. For clarity, we also set $d B_{t}=0$ (in the path but not the value and policy functions). Early on, the consumption path is increasing and below one, as the country accumulates reserves, which it then uses during the sudden stop. The country's incentive to accumulate reserves is very steep initially and less intense once some reserves have been accumulated. Importantly, even though the sudden stop in this example takes place exactly at its expected time, there is a significant consumption drop at the time of the sudden stop, reflecting the imperfect nature of the reserves accumulation self-insurance mechanism.

The next two figures contain the distributions of the main quantities generated by the model, associated to a large number of paths in our economy. Recall that randomness stems from $Y_{t}$ as well as from the transitions in and out of sudden stops. We start with a country that has 0 reserves initially and simulate 


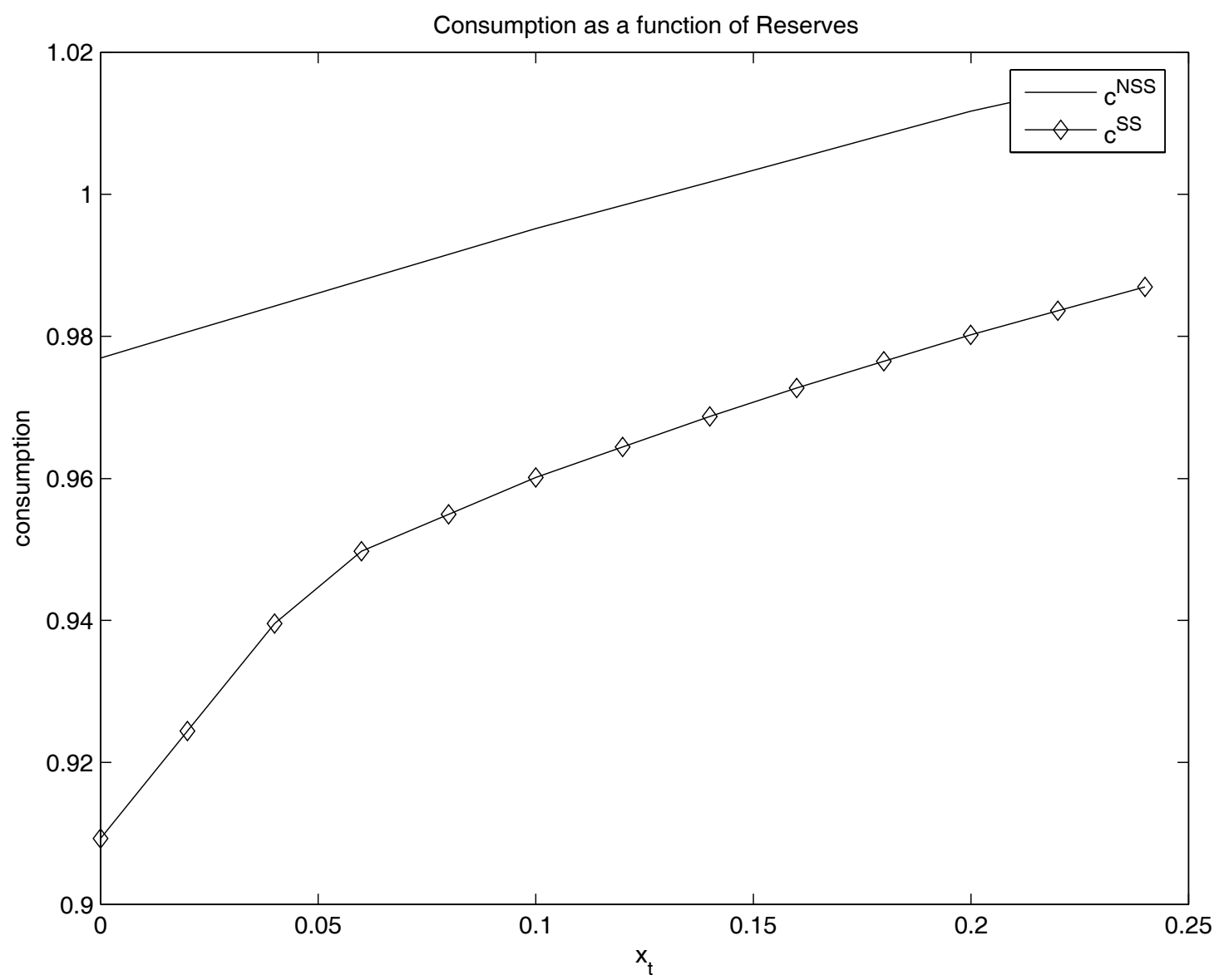

Figure 3: Consumption functions in NSS and SS 

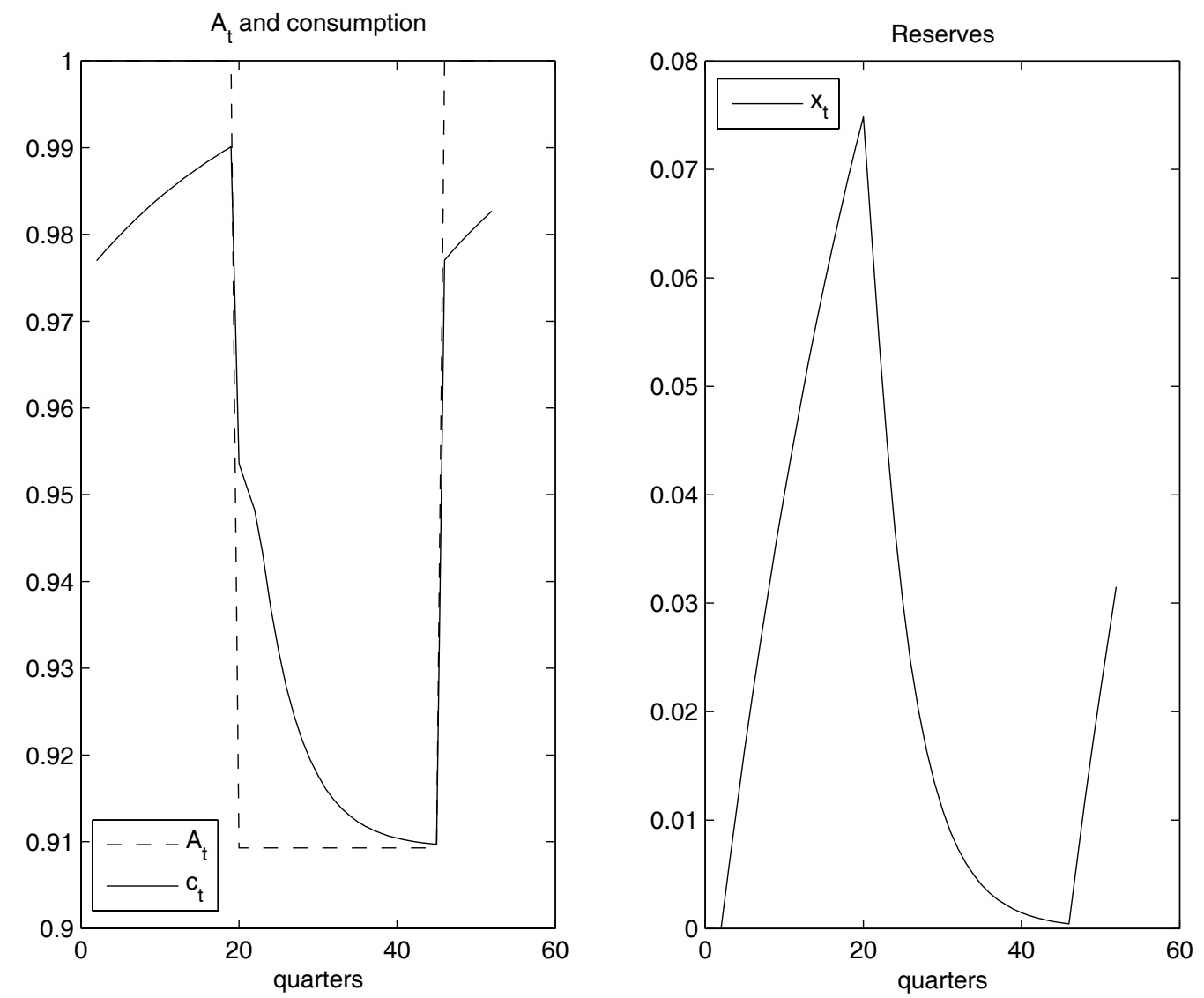

Figure 4: Typical path of consumption and reserves. 


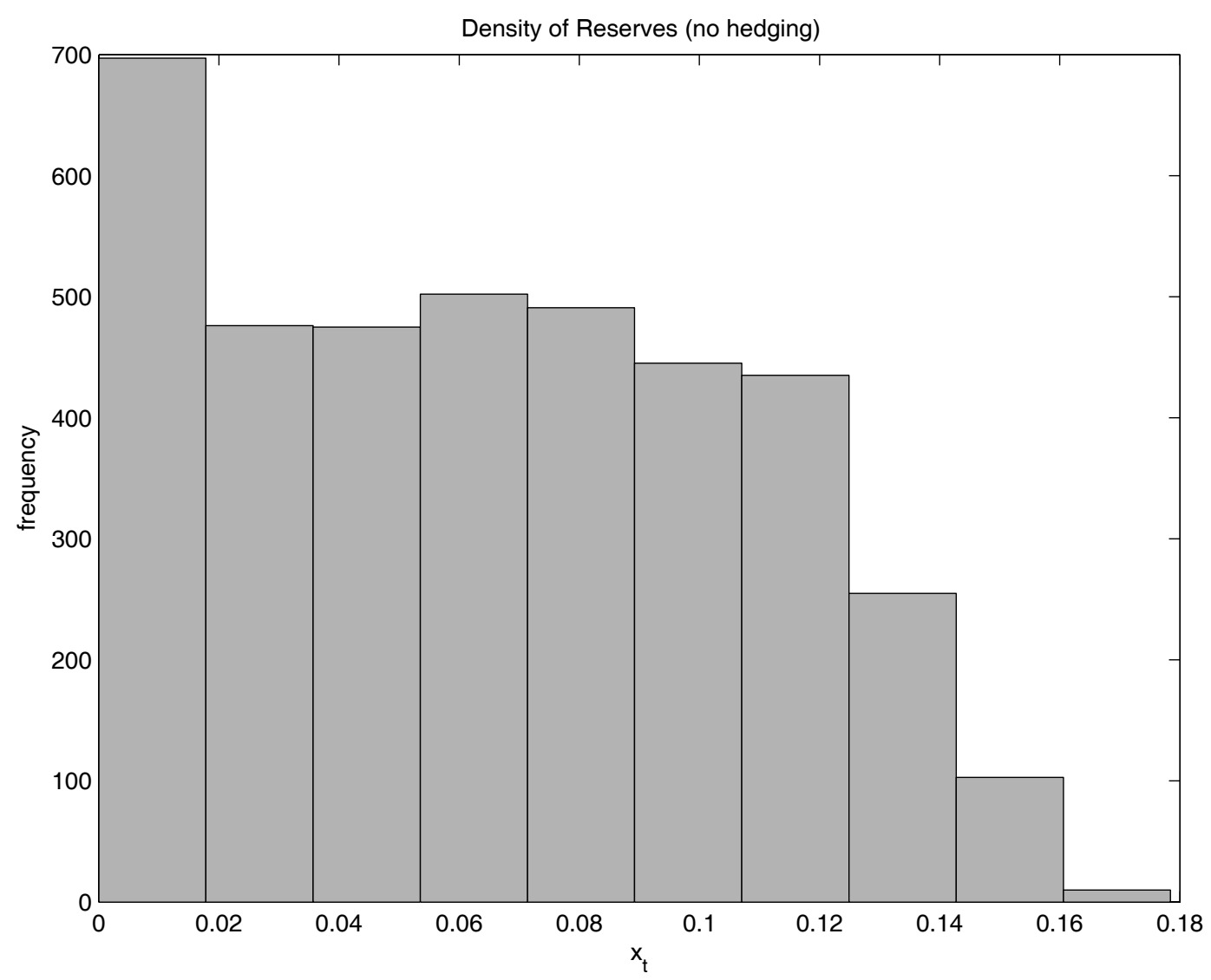

Figure 5: Distribution of Reserves immediately prior to SS.

5000 paths for $T=80$ years, without a transition into development.

Figure 5 displays the histogram of reserves available at the point in which the country enters a sudden stop. The possibility of a sudden stop leads to an accumulation of reserves well above $5 \%$ of available resources during NSS. However it is critical to observe that there a significant mass concentrated at very low levels of reserves. This mass corresponds to those cases where the country has not had the time to accumulate reserves since the previous sudden stop. These early crises are an important source of risk, that reserves are particularly inefficient in dealing with. ${ }^{6}$

Figure 6 displays average consumption during $N S S$ and $S S$. The average difference between consumption is large, around 7 percent. Moreover, often the country simply runs out of reserves during sudden stops and consumption falls by the full extent of the capital flow reversal.

In summary, these results raise the question of whether there are other ways to manage reserves that

\footnotetext{
${ }^{6}$ In practice, countries often deal with this risk by not using reserves very aggressively during sudden stops, which is a clearly suboptimal strategy.
} 

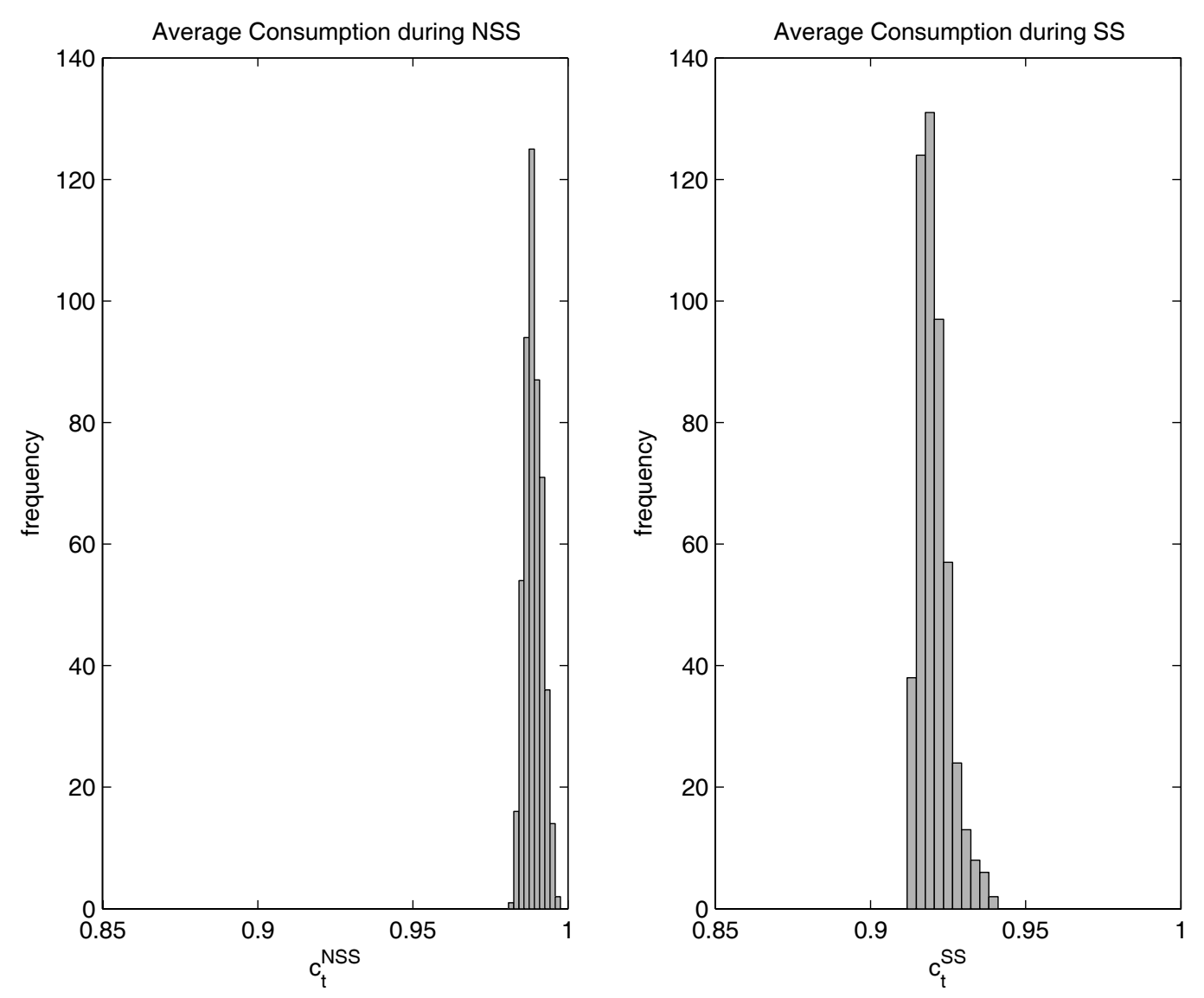

Figure 6: Average consumption during SS and NSS. 
could increase their efficiency and avoid the risk that is associated with the unpredictability of sudden stops. We turn to this question in the next section.

\section{Contingent Instruments}

In practice, how much better could countries do by holding contingent instruments in their reserves? Obviously, the answer to this question depends on the specific factors behind a country's sudden stops and the financial markets available to hedge those specific factors. Our goal here is less ambitious. We simply want to illustrate, in a conservative scenario in terms of information and financial development requirements, the significant potential gains from improving current sudden-stop-risk-management practices by adding contingent instruments that are largely independent of the country's actions and, more generally, of factors exclusively related to emerging markets (and hence not appealing to world capital markets).

\subsection{Portfolio decision}

Before introducing additional assets, let us develop in more detail the events behind a transition into a sudden stop. It is useful to think of this transition in two steps. First, there is a Poisson process with intensity $\chi$ that puts the economy in a "danger zone" at stochastic times $\tau^{D}$. Second, at $\tau^{D}$, the country enters a sudden stop with probability $P(S S=1)$ and avoids it with probability $P(S S=0)=1-P(S S=1)$. It is evident that,

$$
\lambda=\chi P(S S=1)
$$

and that nothing in our analysis up to now is modified by this decomposition of events.

Let us now assume that there is a financial asset with payoff $F_{t}$, that also has the potential to exhibit a jump in "danger zones" $\tau^{D}$, which we denote by $J$. When $J=1$ the asset's payoff exhibit a jump at $\tau^{D}$, while $J=0$ denotes the absence of a jump at such time. Correspondingly, the probability of each event (conditional on $\tau^{D}$ ) are denoted by $P(J=1)$ and $P(J=0)$, respectively. It follows that this jump process also follows a Poisson process with intensity:

$$
\lambda_{J} \equiv \chi P(J=1)
$$

Note that sofar we have not excluded the possibility that the jump in the asset with payoff $F_{t}$ is independent from the transition into the SS $(P(J=1, S S=1)=0))$, although clearly our interest is in the case where:

$$
P(J=1, S S=1)>0
$$

We can now write the risky asset's payoff process as:

$$
d F_{t}=r F_{t} d t+F_{t}\left(J d N_{t}-\chi \bar{J} d t\right)
$$


where $d N_{t}$ is a jump process that takes the value of one at time $\tau^{D}$ and zero otherwise, and $\bar{J}=P(J=1)$ is the mean of $J$. Correspondingly, the asset has a return $\frac{d F_{t}}{F_{t}}$.

In what follows we condition on those times $\tau^{D}$ where we observe either a jump $(J=1)$ and/or a transition into SS. This is without loss of generality, since (as we show shortly) the Bellman equation only depends on those events and not on situations where neither takes place. Hence from now on let us define:

$$
\chi^{*}=\chi(1-P(S S=0, J=0))
$$

which is the hazard rate for observing either a jump in $J$ or a transition into $S S$. An obvious corollary is that there are only three possible outcomes that can take place at those times $(S S=1, J=1)$, or $(S S=0, J=1)$, or $(S S=1, J=0)$. To simplify notation let us define:

$$
\begin{aligned}
& p_{J=1, S S=1}=P(S S=1 \text { and } J=1 \mid S S=1 \text { or } J=1) \\
& p_{S S=1, J=0}=P(S S=1 \text { and } J=0 \mid S S=1 \text { or } J=1) \\
& p_{J=1, S S=0}=P(S S=0 \text { and } J=1 \mid S S=1 \text { or } J=1)
\end{aligned}
$$

To complete the description of the data generating process we assume that once in a sudden stop, the transition out of it, which happens with intensity $\widetilde{\lambda}$, is independent of the jumps in $J$.

The addition of a risky asset with the above properties modifies the analysis in only two respects. First the evolution of reserves (7) becomes

$$
d X_{t}=\left(r\left(X_{t}-\xi_{t} F_{t}\right)-C_{t}+A_{t}\right) d t+\xi_{t} d F_{t}
$$

where $\xi_{t}$ is the amount invested in the risky asset $F_{t}$. It is important to note that the constraint $X_{t} \geq 0$, implies the "short selling" constraint

$$
\xi_{t} \geq-X_{t} / \max (J)
$$

Else, there could be case, where the jump is sufficiently large to violate the non-negativity constraint on $X_{t}$. If $\max (J)=1$ (as we have assumed) we have the portfolio constraint: $\xi_{t} / X_{t} \geq-1$. This means that the risky asset cannot be used to circumvent the borrowing constraint (i.e., it must post full margin).

Second, the optimization problem now involves a portfolio decision. This decision is straightforward in the post-development and sudden stops regions since investing in the asset $F_{t}$ only means adding risk to the country's reserves holdings, without reward in terms of hedging value. Accordingly, the country picks $\xi_{t}=0$ in these regions and the associated Bellman equations of section 2.3 remain unchanged.

Thus the portfolio decision is interesting only in the pre-development non-sudden-stop region (NSS), where the country is preparing itself for a potential sudden stop. Letting:

$$
\widetilde{\xi}_{t}=\frac{\xi_{t}}{\theta^{N S S} Y_{t}}
$$


the Bellman equation in the NSS region is now ${ }^{7}$ :

$$
\begin{aligned}
0= & \max _{c_{t}}\left\{\frac{c_{t}^{1-\gamma}}{1-\gamma}-c_{t} v_{x}^{N S S}\right\}+\left(r x_{t}+1\right) v_{x}^{N S S}+g v^{G}\left(x_{t}\right) \\
& +\max _{\widetilde{\xi}_{t}}\left\{\chi^{*}\left[\sum_{i, j \in\{0,1\}} p_{J=i, S S=j} v^{S S 1\{S S=j\}}\left(x_{t}+\widetilde{\xi}_{t} F_{t} 1\{J=1\}\right)\right]-\widetilde{\xi}_{t} F_{t} \chi^{*} \overline{\bar{J}} v_{x}^{N S S}\right\} \\
& -\left(r+\chi^{*}+g\right) v^{N S S} \\
& +\mu_{Y}\left[(1-\gamma) v^{N S S}-v_{x}^{N S S} x_{t}\right]+\frac{1}{2} \sigma_{Y}^{2}\left(-\gamma(1-\gamma) v^{N S S}+2 \gamma v_{x}^{N S S} x+v_{x x}^{N S S} x^{2}\right)
\end{aligned}
$$

where:

and

$$
v^{S S 1\{S S=j\}}=\left\{\begin{array}{c}
v^{S S} \text { if } S S=1 \\
v^{N S S} \text { if } S S=0
\end{array}\right.
$$

$$
\overline{\bar{J}}=E(J \mid S S=1 \text { or } J=1)
$$

The additional first order condition is:

$$
\sum_{i, j \in\{0,1\}} p_{J=i, S S=j} v_{x}^{S S 1\{S S=j\}}\left(x_{t}+F_{t} \widetilde{\xi_{t}}\right) 1\{J=1\}=\overline{\bar{J}} v_{x}^{N S S}
$$

Notice that this is a standard Euler equation. Defining the rate of return on asset $F_{t}$ as:

$$
R=\frac{1\{J=1\}}{\overline{\bar{J}}}
$$

and using the first order condition for consumption given in (12) we can rewrite (20) as:

$$
E\left[\frac{u^{\prime}\left(C_{\tau^{D+}}\right)}{u^{\prime}\left(C_{\tau^{D-}}\right)} R \mid S S=1 \text { or } J=1\right]=1
$$

where the expectation is taken at time $\tau^{D}$ but without knowledge of which of the three possible combinations of $J$ and $S S$ is about to materialize.

In the extreme case where $p_{J=1, S S=1}=1$, the country has an "ideal" instrument at its disposal and the Euler equation collapses to:

$$
u^{\prime}\left(C_{\tau^{D+}}\right)=u^{\prime}\left(C_{\tau^{D-}}\right)
$$

which implies $C_{\tau^{D+}}=C_{\tau^{D-}}$ and hence there is no consumption drop upon entering a SS. Moreover, one can show that in this case the country has no incentive to accumulate reserves and instead just purchases enough contingent instruments to ensure that $C_{\tau^{D+}}=C_{\tau^{D-}}$.

More generally, both $p_{J=0, S S=1}$ and $p_{J=1, S S=0}$ are positive, which implies that there can be significant injections of resources that do not come with sudden stops, as well as sudden stops with significant consumption drops since the contingent strategy does not yield the resources in a timely manner. In this case, the Euler equation simply balances the risks of these events. Doing so generally requires accumulating non-contingent and contingent reserves, an issue we return to in the empirical implementation below.

\footnotetext{
${ }^{7}$ In the second line $p_{J=0, S S=0}=0$ since we condition on events where either $J=1$ or $S S=1$.
} 


\subsection{Implementation}

The strategy described above assumes that the country and the world capital markets can write a contract that is contingent on a jump in the price of a traded asset. It is not immediately obvious how to write such a contract. In this section we describe a practical way to create a contract that pays 1 if a traded asset exhibits a jump and 0 otherwise. Assume that there exists a state variable with dynamics:

$$
d w_{t}=\mu_{w} d t+\sigma_{w} d B_{t}+J_{w} d N_{t}
$$

and that there exist call and put options that are written on this state variable. Fix first $\Delta, \varepsilon, \varepsilon_{1}>0$ and assume that the country goes long a call option with exercise price $w_{t}+\sigma_{w} \sqrt{\Delta}+\varepsilon$ and short a call option with strike price $w_{t}+\sigma_{w} \sqrt{\Delta}+\varepsilon+\varepsilon_{1}$. Such a scheme pays $\varepsilon_{1}$ if $w_{t+\Delta}>w_{t}+\sigma_{w} \sqrt{\Delta}+\varepsilon+\varepsilon_{1}$ and 0 if $w_{t+\Delta}<$ $w_{t}+\sigma_{w} \sqrt{\Delta}+\varepsilon$. Now suppose that it can be repeated $N$ times, so that $\varepsilon_{1} \rightarrow 0$ and $N \varepsilon_{1} \rightarrow 1$. Then, one obtains in the limit a "digital" option that pays 1 if $w_{t+\Delta}>w_{t}+\sigma_{w} \sqrt{\Delta}+\varepsilon$, and 0 otherwise. Furthermore assume that we take $\Delta$ to 0 , by considering contracts of very small maturity. Then we have a contract that pays 1 if $w_{t^{+}}-w_{t^{-}}>\varepsilon$, i.e. if $w_{t}$ exhibits a discontinuity larger than $\varepsilon$. If the distribution of $J_{w}$ has a positive lower bound then taking $\varepsilon$ to be that lower bound yields a contract that has a payoff of 1 if there is a jump and 0 otherwise. ${ }^{8}$ The payoff of such a strategy would then be equivalent to the payoff of an asset following the process in equation (19) and the analysis of the previous section follows.

It is important to note that the above argument is an approximation argument. We obtain a contract that delivers a payoff conditional on a jump as the limit of trading strategies with existing securities. From a practical perspective, creating a payoff that resembles a digital option from puts and calls is relatively straightforward. Investment banks are often willing to provide quotes for such contracts directly. The more subtle part of the argument is the limit as $\Delta \rightarrow 0$. In the econometric estimations that follow we therefore estimate the "correlation" between a contract that would have a payoff of 1 if $w_{t+\Delta}-w_{t}$ is larger than a fixed amount $\varepsilon$, and $\Delta$ is taken to be a month.

\section{Quantitative Analysis}

Our goal here is not to conduct a thorough search for the optimal risky instrument for specific countries' portfolios. Rather, we seek to illustrate the kind of properties that such instruments ought to have and their implications. With this purpose, we chose the CBOE Volatility Index (VIX). This is a traded index formed from quoted put and call options on the S\&P 500, available since the late 1980s. This index extracts the "implied" volatility from the underlying options. Traders then can take positions in this index to implement

\footnotetext{
${ }^{8}$ If the distribution of the jump has no lower bound then we can still obtain a payoff similar to the above with high enough probability as long as we set the "cutoff" $\varepsilon$ low enough and $\operatorname{Pr}\left(\zeta_{w}>\varepsilon\right)$ is close to 1 .
} 
hedges or speculate. In line with the model, this index is primarily driven by US and not emerging market events. Moreover, as we show below, sudden stops are highly correlated with jumps in the VIX. These two properties are key for useful and potentially liquid SS-hedging instruments.

\subsection{The VIX Process}

Let us postulate a continuous time process of the VIX, described by an Ornstein-Uhlenbeck with jumps:

$$
d \log (V I X)=A(B-\log (V I X)) d t+\sigma_{V I X} d B_{t}+\left(\phi d N_{t}-\lambda_{J} \mu_{\phi} d t\right)
$$

Notice that this process is of the form considered in section 4.2. The first term captures the predictable component of the process, which plays no interesting role in what follows. For simplicity we shall take $A, B, \sigma_{V I X}$ to be constant and $\phi$ to be a normal distribution with mean $\mu_{\phi}$ and standard deviation $\sigma_{\phi}$. The jump process $d N_{t}$ takes the value 1 when a jump takes place and 0 otherwise. The parameter $\lambda_{J}$ denotes the hazard rate for the jump process. Finally, note that the second and third terms in the process are martingale differences.

In estimation, we shall approximate the above process by its discrete time counterpart. The first step is to remove the predictable component, for which we estimate an $\mathrm{AR}(1)$ process for $\log (V I X)$ and focus on the residuals. These residuals are characterized by a mixture of normals:

$$
e_{t}=\mu_{V I X} \Delta+\sigma_{V I X} \sqrt{\Delta} \varepsilon_{t+\Delta}+\phi_{t+\Delta} 1\{J=1\}
$$

with $\mu_{V I X} \approx-\lambda_{J} \mu_{\phi} \Delta$ and $1\{J=1\}$ denotes an indicator that takes the value 1 if a jump takes place between $t$ and $t+\Delta$, and $\left(\varepsilon_{t+\Delta}, \phi_{t+\Delta}\right)$ are i.i.d. Normal:

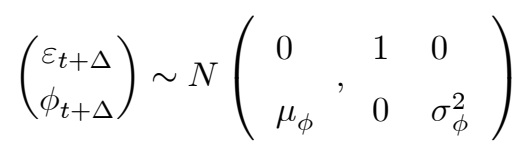

The source of the approximation to the continuous time limit, is that the discrete approximation excludes the possibility of more than one jump in the interval $\Delta$, which seems reasonable if we want to focus on relatively large and infrequent jumps and relatively small time intervals $\Delta$.

Note that (21) can be rewritten as:

$$
e_{t}=\left(1-p_{V I X}\right) N\left(\mu_{V I X} \Delta, \sigma_{V I X}^{2} \Delta\right)+p_{V I X} N\left(\mu_{V I X} \Delta+\mu_{\phi}, \sigma_{V I X}^{2} \Delta+\sigma_{\phi}^{2}\right)
$$

with $p_{V I X}=1-e^{-\lambda_{J} \Delta}$.

In principle, estimation can proceed from this point on along conventional jump-diffusion estimation (see, e.g., Caballero and Panageas (2004)). Instead of following this path, we adopt a strategy that is more 
directly linked to the implementation strategy described in section $4.2 .{ }^{9}$ We assume that the country can obtain contracts that pay off 1 unit if the residual from the $\log (V I X)$ AR1-regression is above 0.259. This is the cutoff that ensures that the type I error of wrongly accepting an observation as a jump is less than $1 \%$ (see the appendix):

$$
\operatorname{Pr}\left(e_{t}>0.259 \mid J=0\right)<0.01
$$

Note that this rule clearly selects the jumps, but there is some residual Type I error associated with the diffusion component of $e_{t}$.

Figure 7 shows the residuals of an an $\mathrm{AR}(1)$ model for $\log (V I X)$. The shaded areas represents those instances when this statistic is above 0.259. The VIX exhibits significant jumps in the early 90's (at the onset of the gulf war) in 1997 (around the Asian crisis), in 1998 (around the Russian/LTCM crisis), after 9/11/2001, and around the beginning of the U.S. corporate scandals and the Argentinean default.

This procedure gives us estimates of the times when the (AR1 residuals of the) VIX experience jumps. With these estimates we turn next to determining the joint occurrence of jumps and transitions into SS for the various countries.

\subsection{Conditional Probabilities}

The final step to be able to assess the benefits of hedging, is to find an estimate of the degree of "correlation" between jumps in the VIX and transitions into SS.

We use a Bayesian procedure to estimate the parameter

$$
P(S S \mid J)=\frac{p_{J=1, S S=1}}{p_{J=1, S S=0}+p_{J=1, S S=1}}
$$

, as described in detail in the appendix. In essence, we start by marking the dates associated with jumps in the VIX as determined above. Effectively we are conditioning on this information. We then use the paths associated with the repetitions of the Gibbs sampler to identify the dates in which individual countries entered into a SS (applying the parameters estimated for the long annual sample on quarterly data for the 1990s). Second, we update the (uniform - uninformative) prior by counting the number of times that the VIX jumps and the country transits from an NSS state into an SS state. We pool all countries together as in section 3.1 in order to exploit the cross sectional dimension of the panel. Third, we condition on the NSS states and count the total number of jumps in the VIX during such states. This allows us to update the probability that within each quarter the VIX jumps (we denote this probability as $P(J)$ ). We also count the number of times that a given country transits into SS. Fourth, we obtain a (beta) posterior distribution for $P(S S \mid J)$ and $P(J)$ from which we take a separate draw for each repetition of the sampler. To check

\footnotetext{
${ }^{9}$ If anything, this procedure is biasing the results towards finding a lower "correlation", because it introduces the potential of type I error compared to Bayesian filtering.
} 


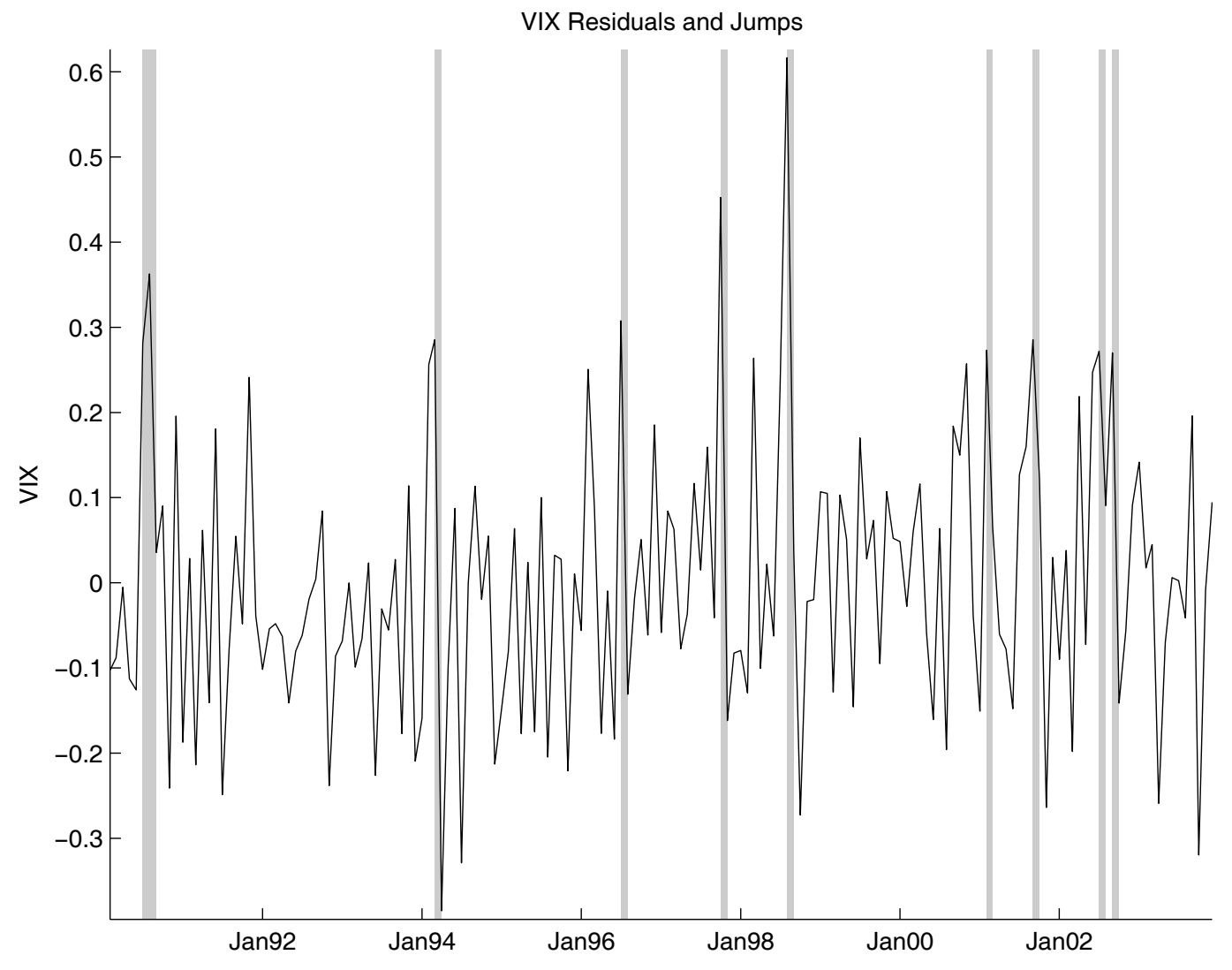

Figure 7: VIX residuals. The grey areas correspond to instances where the VIX residual is above the jump-cutoff. 


\begin{tabular}{lccccccc}
\hline \hline & Average & Std Dev & $5 \%$ & $25 \%$ & $50 \%$ & $75 \%$ & $95 \%$ \\
\hline Beta & 0.159 & 0.032 & 0.109 & 0.137 & 0.158 & 0.180 & 0.215 \\
Empirical & 0.155 & 0.006 & 0.145 & 0.151 & 0.155 & 0.159 & 0.163 \\
\hline \hline
\end{tabular}

Table 4: $\operatorname{Pr}(J)$ Pooled Estimator, quarterly data 1990-2003. Bayesian Updating and Empirical Distribution.

\begin{tabular}{lccccccc}
\hline \hline & Average & Std Dev & $5 \%$ & $25 \%$ & $50 \%$ & $75 \%$ & $95 \%$ \\
\hline Beta & 0.236 & 0.096 & 0.095 & 0.165 & 0.226 & 0.296 & 0.413 \\
Empirical & 0.211 & 0.040 & 0.150 & 0.190 & 0.200 & 0.238 & 0.286 \\
\hline \hline
\end{tabular}

Table 5: $\operatorname{Pr}(S S \mid J)$ Pooled Estimator, quarterly data 1990-2003. Bayesian Updating and Empirical Distribution.

the influence of this prior on our estimates we also report directly the distribution of the ratio of joint occurrences $(N S S \rightarrow S S=1$ and $J=1)$ to total occurrences $(N S S \rightarrow S S=1$ or $J=1)$. Similarly, we also report the distribution of the ratio of total occurrences of jumps only $(J=1)$ to total occurrences of either $\operatorname{event}(N S S \rightarrow S S=1$ or $J=1)$. We label this the "empirical" distribution.

In tables 4 and 5 we report the results of this estimation. Table 4 contains the estimates for $P(J)$ and table 5 contains estimates for $P(S S \mid J)$. The top line in both tables includes statistics for the posterior distribution obtained through the formal Bayesian procedure described above. The bottom line contains the "empirica" distribution, which is only given for an intuitive "robustness" check on the influence of the uniform prior on the posterior location parameters like the mean and the median.

From this table one can obtain the implied intensity of a jump in the VIX as:

$$
\lambda_{J}=-4 \log (1-\overline{P(J)})=0.69
$$

where $\overline{P(J)}$ denotes the mean of the posterior distribution of $P(J)$.

Now we can identify all the key parameters of the joint VIX and SS process, which can be recovered from the following four relations:

$$
\begin{aligned}
\lambda_{J} & \equiv \chi^{*}\left(p_{J=1, S S=1}+p_{J=1, S S=0}\right) \\
\lambda & \equiv \chi^{*}\left(p_{J=1, S S=1}+p_{J=0, S S=1}\right) \\
\operatorname{Pr}(S S \mid J) & =\frac{p_{J=1, S S=1}}{p_{J=1, S S=0}+p_{J=1, S S=1}} \\
1 & =p_{J=1, S S=1}+p_{J=1, S S=0}+p_{S S=1, J=0}
\end{aligned}
$$

Since we have obtained estimates for $\lambda_{J}, \lambda$ (from section 3.1) and $\operatorname{Pr}(S S \mid J)$ from the above table, we 


\begin{tabular}{cccccc}
\hline \hline$\gamma$ & 8 & $\lambda$ & 0.211 & $\operatorname{Pr}(S S=1$ and $J=1)$ & 0.221 \\
$\delta$ & 0.04 & $\tilde{\lambda}$ & 0.148 & $\operatorname{Pr}(S S=1$ and $J=0)$ & 0.063 \\
$r$ & 0.04 & $\eta$ & $(0.07,0.1,0.14)$ & $\operatorname{Pr}(S S=0$ and $J=1)$ & 0.716 \\
$\mu$ & 0.018 & $g$ & 0.025 & $\chi^{*}$ & 0.743 \\
$\sigma$ & 0.05 & & & $\lambda_{J}$ & 0.696 \\
\hline \hline
\end{tabular}

Table 6: Parameters used in Simulations.

\begin{tabular}{lccccccc}
\hline \hline & Average & Std Dev & $5 \%$ & $25 \%$ & $50 \%$ & $75 \%$ & $95 \%$ \\
\hline Beta & 0.636 & 0.16122 & 0.354 & 0.526 & 0.645 & 0.759 & 0.883 \\
Empirical & 0.672 & 0.088 & 0.556 & 0.625 & 0.667 & 0.714 & 0.857 \\
\hline \hline
\end{tabular}

Table 7: $\operatorname{Pr}(J \mid S S)$ Pooled Estimator, quarterly data 1990-2003. Bayesian Updating and Empirical Distribution.

can solve for the four unknowns $p_{J=1, S S=1}, p_{S S=1, J=0}, p_{J=1, S S=0}$ and $\chi^{*}$. Table 6 summarizes the resulting parameters that we use in the simulation in the next section.

Finally, we also obtain estimates $\operatorname{Pr}(J \mid S S)$, which report in Table 7 . They indicate that the probability of observing a "jump" conditional on a transition to SS is about 70 percent. Which means that with very high probability the contract that we consider delivers payoffs exactly in the states where inflows are needed the most.

\subsubsection{Implications}

In this section we calibrate the model with the same parameters as the ones used in section 3.2. To provide a base case scenario we use the median $p_{J, S S}$. Using the same $\lambda$ as in section 3.2 and the $\lambda_{J}$ obtained in 5.1 we can determine $\chi^{*}, p_{S S=1, J=0}$ and $p_{J=1, S S=0}$ from the procedure described above and solve the model numerically.

For the same realizations in section 3.2, plus the corresponding realizations of simulated VIX processes, panel (a) in Figure 8 reports the difference in reserves at the time of the sudden stop between the contingent and non-contingent strategies, normalized by the average of the latter. The empirical mean of this distribution is close to 30 percent. This is a direct consequence of the efficiency of hedging compared to non-contingent reserve accumulation. By adopting the optimal hedging strategy the country manages to transfer more resources towards the states it is concerned with, namely the onset of a $S S$.

However, the above distribution and its mean underestimates the benefits of hedging. It is apparent that it is much worse to have less reserves when there is little of them to start with (which is when the hedging strategy typically dominates by a wide margin), than to have fewer reserves when the country has had plenty 

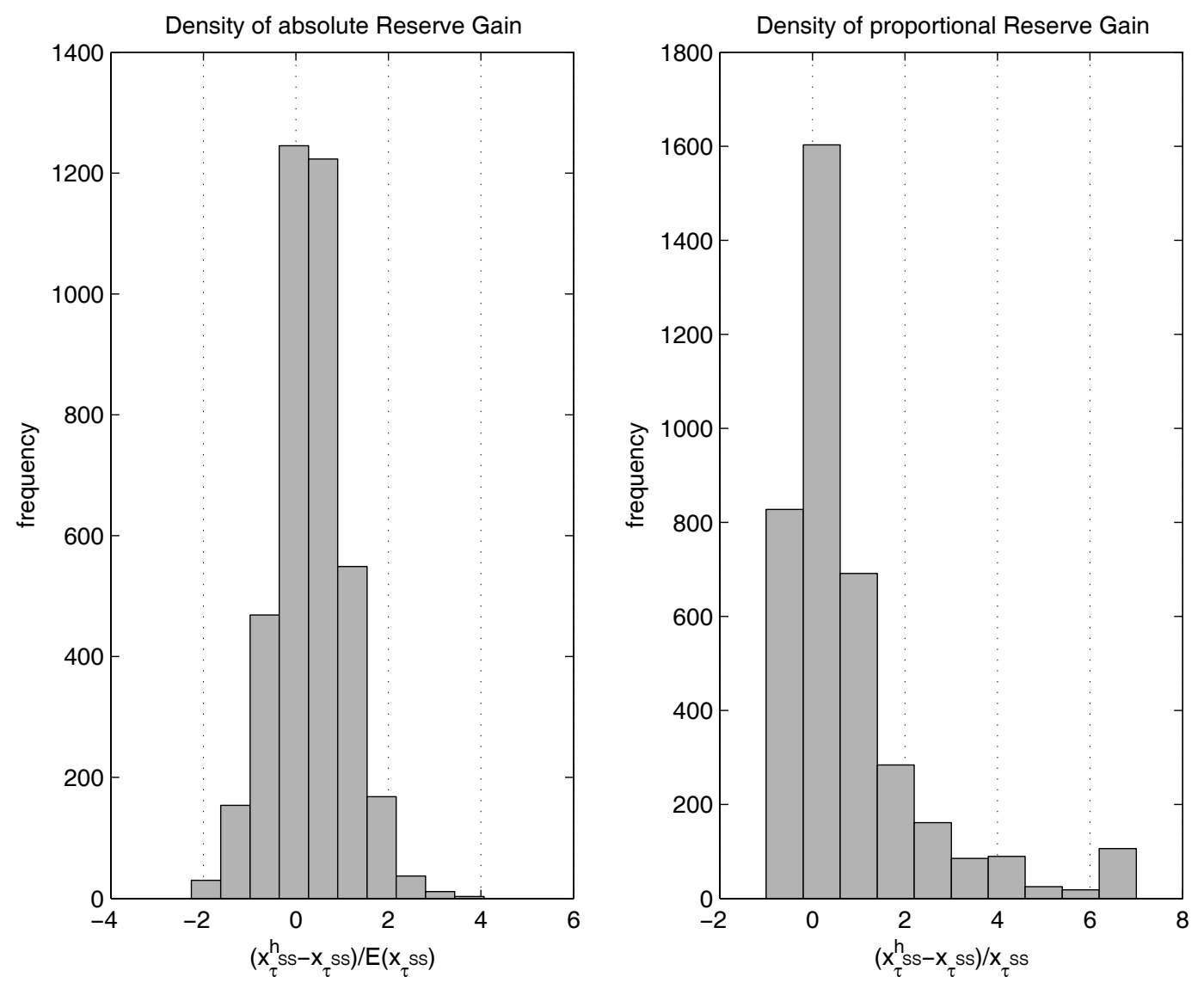

Figure 8: Reserves Gain (in absolute and proportional terms) 


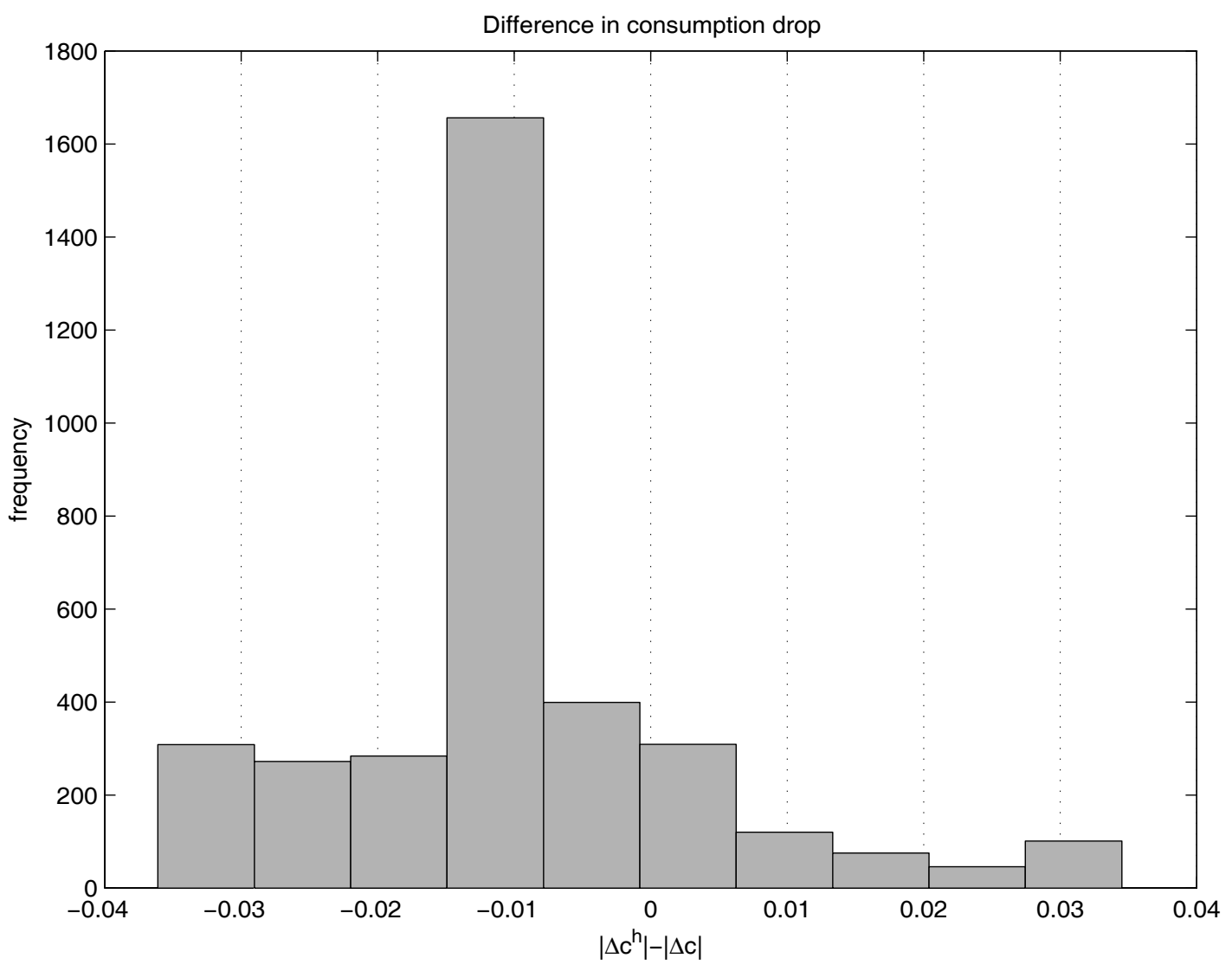

Figure 9: Difference in the magnitude of consumption drop upon entering the SS 


\begin{tabular}{lccc}
\hline \hline & $\eta=0.1$ & $\eta=0.14$ & $\eta=0.07$ \\
\hline Mean Level of Reserves & 0.065 & 0.137 & 0.019 \\
Median Level of Reserves & 0.064 & 0.132 & 0.019 \\
Mean Reserves (first quartile) & 0.011 & 0.022 & 0.0034 \\
Mean Reserves (fourth quartile) & 0.122 & 0.263 & 0.0356 \\
Median (normalized) reserves gain & 0.293 & 0.047 & 1.658 \\
Median (normalized) reserves gain (first quartile) & 2.797 & 1.265 & 9.422 \\
Median (normalized) reserves gain (fourth quartile) & -0.058 & -0.130 & 0.801 \\
Absolute value of Median difference in consumption drop & 0.010 & 0.010 & 0.022 \\
Absolute Value of Median difference (75th Percentile) & 0.015 & 0.013 & 0.027 \\
Absolute Value of Median difference (25th Percentile) & 0.007 & 0.006 & 0.015 \\
Median Portfolio & 0.700 & 0.562 & 0.776 \\
\hline \hline
\end{tabular}

Table 8: Performance of hedging strategies

of time to prepare for the sudden stop (which is when the unhedged strategy may do better). To capture this important asymmetry, panel (b) in figure 8 plots the histogram for:

$$
\Delta x^{R}=\frac{x_{\tau S S}^{h}-x_{\tau S S}}{x_{\tau} S S}
$$

This expression gives the relative reserves gain. As might be expected the distribution of $\Delta x^{R}$ is right skewed. The hedge performs especially well when the country has not had the time to accumulate non-contingent reserves. An alternative way of making the same point is seen in the first column of Table $8(\eta=0.1)$, for the row labelled "median (normalized) gain (first quartile)," which shows that the median gain from the contingent strategy when reserves are low is close to 300 percent (since the mean of reserves is 0.011 , this implies an absolute gain in reserves around 3 percent of $\left.\theta^{N S S} Y\right)$.

In terms of the drop of consumption at the instant of the sudden stop, the above difference translate into a median difference that exceeds 1 percent of maximum NSS-consumption and, as shown in Figure 9, has significant mass at drop-differentials four times as large as that.

Figure 10 displays the notional amount of contingent contracts normalized by reserves as a function of $x_{t}$. Two observations emerge from this figure: First, as a percentage of $x_{t}$, the amount invested in contingent contracts declines. Second, the country enters a large number of these contracts. For instance when $x=0.05$, the country enters contracts that equal total reserves. And when $x$ is as large as 0.15 , the contracts still exceed 30 percent of reserves.

In terms of dynamics, these two observations mean that the country should first build the contingent part of the portfolio aggressively, adding non-contingent reserves only gradually. The reason for this strategy 


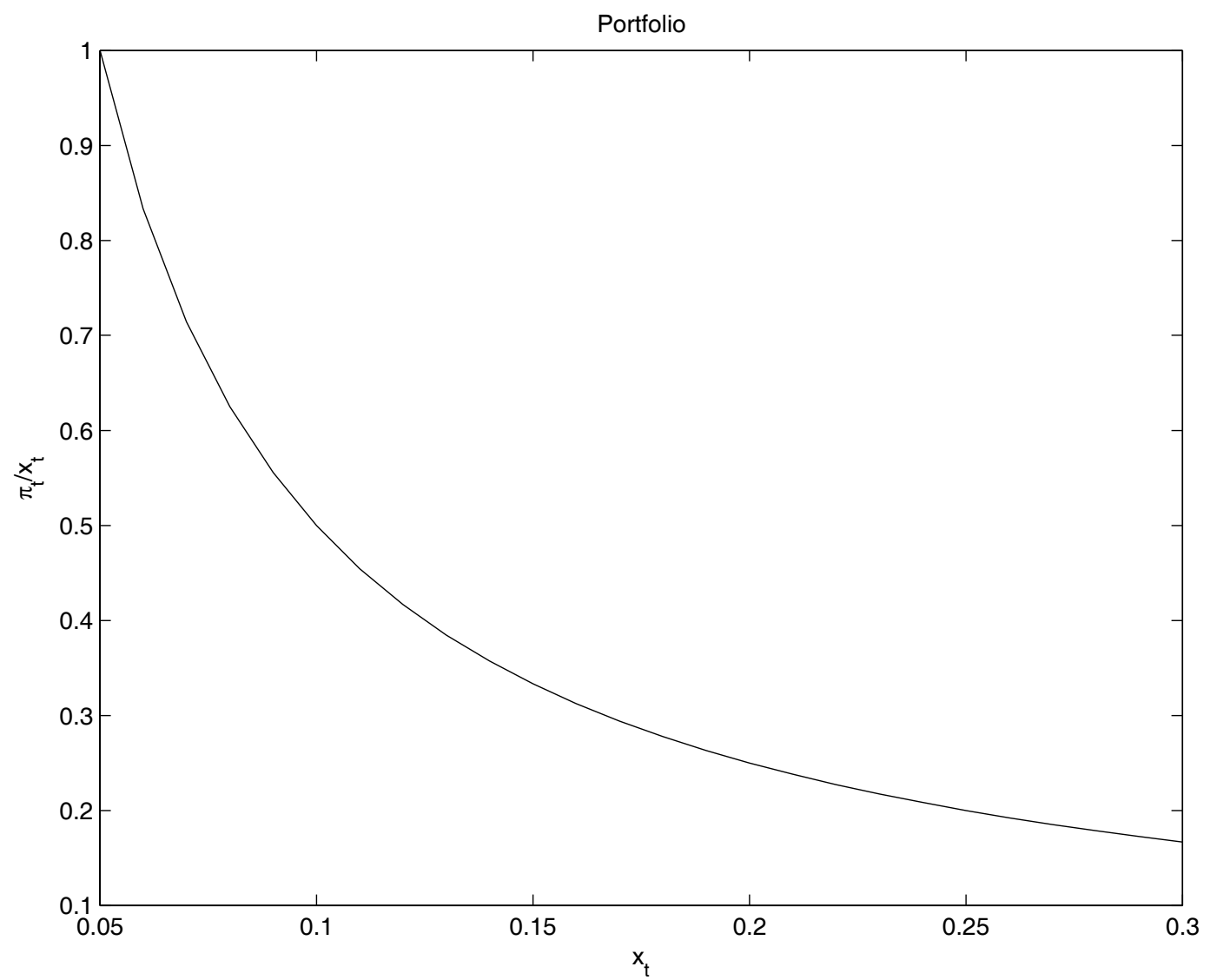

Figure 10: Notional amount invested in contingent instruments normalized by reserves. 
is that the country is particularly concerned about the worst possible event. That is, is to be found without resources at the time of a sudden stop. When the stock of reserves is small, the best chance of avoiding this worst-event is by getting a large contingent payment at the time of the sudden stop. When the stock of reserves is large, the worst-event is more likely to take place if the country overcommits to contingent contracts and these do not deliver, than by holding a large amount of non-contingent reserves.

These features also have implications across different configurations of parameters. Going back to Table 8 , the columns report results for three scenarios that only vary in the size of the sudden stop. In addition to the benchmark scenario, we report results for the 25 and 75 percentiles of our estimated $\eta \mathrm{s}$. The results suggest that as countries improve along dimensions that reduce the size of sudden stop, ${ }^{10}$ they should not only reduce their reserves accumulation but also reallocate their portfolios toward more contingent instruments.

\section{Debt and Contingent Liability Management}

Let us now relax the constraint $W=0$ and allow the country to borrow from WCM, so that:

$$
X_{t} \geq-W_{t} \text { a.s. }
$$

for some process $W_{t}>0$. This mean that net assets with $W C M$ as the counterparty $\left(X_{t}\right)$ can potentially turn negative. In principle $W_{t}$ could be any process. However, it is most reasonable to assume that $W_{t}$ is proportional to income:

$$
X_{t} \geq-w Y_{t}
$$

The point of this short section is that the essence of our analysis of reserves remains valid here, now for net assets (or liabilities) management.

In fact, without further modifications the analysis is identical to that in the previous sections since the country would behave as if $w=0$. The reason for this is that for any $X_{t}<0$, there is a path of the diffusion in income that would lead to negative consumption. The standard approach to remove this unpleasant implication is to "complete" the market by introducing a new asset $S_{t}$ with payoffs:

$$
\frac{d S_{t}}{S_{t}}=r d t+\sigma_{S} d B_{t}
$$

where $d B_{t}$ is the same Brownian motion that drives the income process. ${ }^{11}$

By adding this asset to its portfolio, the country removes the possibility of a perverse path and is now willing to borrow from WCM. ${ }^{12}$

\footnotetext{
${ }^{10}$ For example, the estimate of $\eta$ we report for Chile corresponds to an average of 0.11 and 0.08 for the 1980s and 1990s, respectively.

${ }^{11}$ See Duffie and Huang (1986).

${ }^{12}$ We view the introduction of this asset as a technical device to make the substantive point we wish to address here rather
} 


$$
R_{t}=X_{t}+w Y_{t} \geq 0
$$

The presence of $S$ in the country's portfolio allows it to effectively remove the diffusion component of the income process but this changes in no substantive way our analysis. In particular, if there are no sudden stops, and if $w$ is not too large, the country never wants to increase $X$. But, more importantly, if there are sudden stops, it is optimal for the country to rely on contingent instruments correlated to the sudden stops rather than just on ex-ante slack in the constraint 25.

In summary, the implications and recommendations stemming from our analysis carry beyond the problem of reserves management to that of external assets and liability management. A country that experiences sudden stops benefits from indexing its assets and liabilities to variables that are correlated with these sudden stops. Public debt, for example, should have coupons and principal that fall as the VIX (or similar variables) crosses certain critical thresholds.

\section{$7 \quad$ Final Remarks}

Emerging market economies hold levels of international reserves that greatly exceed the levels held by developed economies (relative to their size). This would seem paradoxical given that, unlike the latter, the former face significant financial constraints with much of their growth ahead of them. The paradox disappears once these greater financial constraints also become an important source of volatility, which countries seek to smooth. This is the context we have modelled, analyzed, and began to assess quantitatively.

Once such perspective is adopted, one must ask whether current practices, consisting primarily in accumulating non-contingent reserves, are the best countries can or should aim to do. How effective are reserves in smoothing the impact of sudden stops unrelated to a country's actions? How much of them should be accumulated? How fast? Are there potentially less costly financial mechanisms to deal with capital flow volatility? Who would be the natural counterpart for these mechanisms? How are these mechanisms limited by financial and collateral constraints?

Our framework provides aspects of an answer to each of these questions: Even if optimally managed, reserves offer limited insurance, should be accumulated at a slower pace and used more aggressively during sudden stops, than is being done by prudent emerging market economies. However, the most important

than as a claim that such assets exist in reality. An alternative mechanism to make the same substantive point, is simply to assume that there is a lower bound for $Y$. One of the main reasons the literature prefers the somewhat artificial "complete markets" solution is that in such case one can use the very general methods of El Karoui and Jeanblanc Pique (1998), and He and Pages (1993) to solve the country's post-development problem. As El Karoui and Jeanblanc Pique (1998) demonstrate, solving problems of this kind amounts to solving the complete markets problem and subtracting the value of a perpetual American option on the difference between the constraint-free net asset process and the constraint. 
message of the paper is that there are potential insurance contracts, credit lines and hedging markets, that could significantly reduce the cost and improve the efficiency of mechanisms to smooth sudden stops. The natural counterpart of these instruments and contracts are not the regular emerging market specialist investors but the world capital markets at large. This is an important consideration to have in mind when designing such instruments. The much touted GDP-indexed and peso-bonds, for example, while a natural and useful instrument to trade with specialists (in fact, they do in our model) are unlikely to appeal to the broad markets. Non-contingent reserves have a place as well, since in practice hedges are unlikely to be perfect, and overcommitting to an imperfect hedge comes with its own risks. It is clear, nonetheless, that there are enough verifiable and contractible global variables that are significantly correlated with sudden stops and should form the basis for a better contingent strategy. Importantly, the very same financial constraints that are behind these countries' troubles, limit the type and amount of insurance and hedging strategies these countries can engage in. In particular, since sudden stops are mostly times when specialists are constrained as well, the strategies must be such that require little credibility and commitment on the country side. This means, essentially, policies and investments that are paid (or collateralized) up-front rather than simple swaps of future contingencies. 


\begin{tabular}{|ll|}
\hline Series & Source \\
\hline Nominal GDP $(G D P)$ & $\begin{array}{l}\text { World Development Indicators } \\
\text { (quarterly and annual) }\end{array}$ \\
\hline CPI $(P)$ & IFS \\
& (quarterly and annual) \\
\hline Nominal Exports $\left(P_{X} X\right)$ & World Development Indicators and IFS \\
(local currency) & (quarterly and annual) \\
\hline Nominal Imports $\left(P_{M} M\right)$ & World Development Indicators and IFS \\
(local currency) & (quarterly and annual) \\
\hline $\begin{array}{l}\text { Real Exports }(X) \\
\text { (local currency) }\end{array}$ & World Development Indicators \\
\hline Real Imports $(M)$ & (annual) \\
(local currency) & World Development Indicators \\
\hline Nominal Capital Flows $(C F)$ & (annual) \\
(dollars) & IFS \\
\hline Nominal Exchange Rate $(E)$ & (quarterly and annual) \\
\hline Net Factor Payments $(N F P)$ & World Development Indicators and IFS \\
(dollars) & (quarterly and annual) \\
\hline
\end{tabular}

Table 9: Data used in the construction of $\psi$.

\section{Appendix}

\section{A Data}

\section{The Data}

The VIX is publicly available from the CBOE on a daily frequency. For an introduction to the construction of this index, see CBOE (2003). We used a monthly frequency in order to smooth spurious daily volatility.

For the construction of $\psi_{i t}$, we used data from the World Bank's World Development Indicators Database, and from the International Monetary Fund's International Financial Statistics (IFS). Table 9 presents a list of the variables and corresponding sources.

While in the model $\psi$ is straightforward, in the data its computation is more cumbersome since there are multiple goods, exchange rate fluctuations, intermediate goods, and so on. All our steps below are aimed at isolating in $\psi$ the component of external resources and income which is transitory in nature. For this, we 
let:

$$
\psi_{t}=\frac{\left(\theta_{t}-1\right) Y}{Y}=\frac{\frac{E_{t} C F_{t}}{P_{M, t}}+\left[\left(\frac{P_{X, t} X_{t}}{P_{M, t}}-0.5 X_{t}\right)\right]^{\text {Cycle }}}{\left[N_{t}\right]^{\text {Trend }}+\left[\left(\frac{P_{X, t} X_{t}}{P_{M, t}}-0.5 X_{t}\right)\right]^{\text {Trend }}},
$$

where $N$ and $X$ correspond to real nontradables and exports; $P_{X}$ and $P_{M}$ to export and import prices in local currency; and $E$ and $C F$ to the nominal exchange rate and capital flows.

Real nontradables are constructed from:

$$
N_{t}=\frac{1}{P_{N, t}}\left(G D P_{t}-\left(P_{X, t}-0.5 P_{M, t}\right) X_{t}\right)
$$

where GDP is the country's GDP, $P_{N, t}$ is the price of nontradables approximated by the local CPI, and the term $0.5 P_{M, t}$ removes a proxy for intermediate inputs in export-production. The expression $\left(\frac{P_{X, t} X_{t}}{P_{M, t}}-0.5 X_{t}\right)$ captures the terms of trade effect.

We decompose between trends and cycles using a standard Hodrick-Prescott filter; extending the series as much as we could in order to reduce the effect of the end-of-series bias in this procedure. We applied the filter the log of the corresponding variable.

In summary, the denominator in equation (26) measures the average (trend level) of total income and resources, while the numerator attempts to capture the cyclical component of external resources.

The Sample

We work with a sample of six developing countries/emerging markets: Chile, Colombia, Indonesia, Malaysia, Mexico, Thailand, and Turkey. We chose them from the list of countries in Calvo, Izquierdo and Mejia (2004) plus Malaysia. However, since their sample is for the 1990s only andwe needed a longer time series dimension (we used data from 1983 to 2003), we dropped all the countries that either were closed during the 1980s, or had primarily domestic macroeconomic problems, or did not have complete data. Our marginal drop was Korea, for which we did not have good deflators. However, when we re-estimated our model with Korea included (using only capital flows data divided by nominal GDP for Korea ), our results remained essentially unchanged.

We made one adjustment to our construction of $\widetilde{\psi}_{i t}$. In the case of Chile, the cycle around the debt crisis is significantly larger than that of the 1990s. Thus in a first stage we standardized the 1980s and 1990s in order to estimate the hidden-states and the transition probabilities. After that, we inverted the standardization to recover the means of each state in each sub-sample.

Finally, we constructed quarterly series for $\widetilde{\psi}_{i t}$ using a related series approach with quarterly data on capital flows. We restrict the average of the quarterly values to be equal to the annual figure we computed directly using equation (26). Unfortunately, we lack quarterly data for the capital flows for all countries in the 1990-2003 period. To solve this problem we use a linear (or a quadratic) interpolation method to obtain 
quarterly series for the years when we lack quarterly data. Although the point estimates do not change much if we only use interpolation methods, the timing of the event matters for the synchronization between sudden stops and the VIX, so we use as much information from the quarterly capital flows as possible in order to preserve the timing of the joint events. We use interpolation methods for the following countries (dates are shown in parenthesis): Chile (1990), Colombia (1990-1995), and Malaysia (1990-1998).

Reserves

As a reference, the next table shows reserves, reserves net of short term, and total external liabilities for the six economies we study.

\section{B Details on the econometric procedure of Sections 3.1, 5.1, 5.2.}

Section 3.1: To estimate the process described in this section we apply a Bayesian methodology, by using a Gibbs Sampler. The Gibbs Sampler is by now a standard methodology in estimating models involving hidden states (See Kim and Nelson (1999) for an introductory treatment). The basic idea is to exploit knowledge about the conditional distribution of one parameter at a time (fixing all the others) to construct the joint posterior distribution of all parameters.

We modify the basic model that Kim and Nelson (1999) present by pooling all the countries into a single sample. We allow all parameters of the model to differ across countries. However, in order to obtain precise estimates we assume that the transition probabilities into and out of a sudden stop are the same across countries. Moreover, we assume that the joint probability of a jump in the VIX and a simultaneous transition into a sudden stop is common across countries.

The first step of the procedure is to fix a set of initial parameters $\Phi=\left\{\psi_{i}^{N S S}, \psi_{i}^{S S}-\psi_{i}^{N S S}, \sigma_{e, i}^{N S S}, \sigma_{e, i}^{S S}\right.$, $p(N S S \rightarrow S S), p(S S \rightarrow N S S)\}$ and then determine the posterior probabilities that a particular realization of $\widetilde{\psi}_{i t}$ was drawn from the first $(N S S)$ or the second $(S S)$ distribution. To do that we run a standard Hamilton $(1989,1990)$ type filter as described in Kim and Nelson (1999). This allows us to determine a sequence of posterior probabilities that a given realization of the data was drawn from the second $(S S)$ normal distribution. We repeat this process for each country separately and obtain one sequence per country. We shall denote this as $\operatorname{Pr}\left(S S=1 \mid \widetilde{\psi}_{i} ; \Phi\right)$. In the next step we draw an (artificial) sample of 1's and 0's from these posterior probabilities. We use the convention that 1 corresponds to a Sudden Stop and 0 to NSS.

In the next step we take these 1's and 0's as given. Effectively this allows us to proceed as if we knew whether each economy is in $S S$ or not at a given point in time. Then we use this information to determine the posterior distributions of the elements of $\Phi$. Once again we do this in steps as described in Kim and Nelson (1999). We start with determining the posterior distribution of $\left\{\psi_{i}^{N S S}, \psi_{i}^{S S}-\psi_{i}^{N S S}, \sigma_{e, i}^{N S S}, \sigma_{e, i}^{S S}\right\}$ first: To facilitate the updating we use conjugate priors: a) a beta prior for $p(N S S \rightarrow S S), p(S S \rightarrow N S S)$ 


\begin{tabular}{|c|c|c|c|c|c|}
\hline & & Res & $\begin{array}{c}\text { Res } \\
\text { - ST Debt }\end{array}$ & $\begin{array}{c}\text { Res - ST Debt } \\
+ \text { Priv Res }\end{array}$ & $\begin{array}{c}\text { Total Ext } \\
\text { Debt }\end{array}$ \\
\hline \multirow[t]{4}{*}{ Chile } & Avg & $20.6 \%$ & $15.3 \%$ & $15.0 \%$ & $46.5 \%$ \\
\hline & Median & $21.5 \%$ & $16.3 \%$ & $12.6 \%$ & $45.7 \%$ \\
\hline & Max & $22.7 \%$ & $21.0 \%$ & $29.1 \%$ & $63.4 \%$ \\
\hline & Min & $15.4 \%$ & $4.3 \%$ & $-5.0 \%$ & $30.3 \%$ \\
\hline \multirow[t]{4}{*}{ Colombia } & Avg & $11.3 \%$ & $6.1 \%$ & $1.5 \%$ & $36.6 \%$ \\
\hline & Median & $10.0 \%$ & $6.5 \%$ & $2.1 \%$ & $39.1 \%$ \\
\hline & Max & $16.6 \%$ & $10.8 \%$ & $6.4 \%$ & $44.9 \%$ \\
\hline & Min & $8.4 \%$ & $2.4 \%$ & $-3.2 \%$ & $27.1 \%$ \\
\hline \multirow[t]{4}{*}{ Mexico } & Avg & $5.5 \%$ & $0.1 \%$ & $-6.5 \%$ & $28.8 \%$ \\
\hline & Median & $5.6 \%$ & $0.2 \%$ & $-6.7 \%$ & $25.6 \%$ \\
\hline & Max & $7.2 \%$ & $3.6 \%$ & $2.3 \%$ & $43.4 \%$ \\
\hline & Min & $2.7 \%$ & $-4.9 \%$ & $-18.6 \%$ & $20.4 \%$ \\
\hline \multirow[t]{4}{*}{ Indonesia } & Avg & $11.3 \%$ & $7.1 \%$ & $6.9 \%$ & $22.5 \%$ \\
\hline & Median & $7.4 \%$ & $4.8 \%$ & $4.7 \%$ & $17.4 \%$ \\
\hline & $\operatorname{Max}$ & $19.8 \%$ & $15.8 \%$ & $17.4 \%$ & $44.4 \%$ \\
\hline & Min & $4.7 \%$ & $1.9 \%$ & $-0.7 \%$ & $13.3 \%$ \\
\hline \multirow[t]{4}{*}{ Malaysia } & Avg & $29.4 \%$ & $-4.8 \%$ & $-9.1 \%$ & $187.7 \%$ \\
\hline & Median & $28.6 \%$ & $-7.7 \%$ & $-12.1 \%$ & $186.2 \%$ \\
\hline & Max & $42.3 \%$ & $24.3 \%$ & $24.6 \%$ & $237.2 \%$ \\
\hline & Min & $19.6 \%$ & $-25.6 \%$ & $-35.9 \%$ & $147.4 \%$ \\
\hline \multirow[t]{4}{*}{ Thailand } & Avg & $21.3 \%$ & $3.6 \%$ & $-7.8 \%$ & $56.4 \%$ \\
\hline & Median & $20.8 \%$ & $3.3 \%$ & $-3.7 \%$ & $58.2 \%$ \\
\hline & $\operatorname{Max}$ & $28.0 \%$ & $18.6 \%$ & $21.9 \%$ & $93.8 \%$ \\
\hline & Min & $14.1 \%$ & $-6.8 \%$ & $-36.1 \%$ & $32.9 \%$ \\
\hline
\end{tabular}

Table 10: Debt and Reserves: 1990-2002 (\% of GDP) Res: Total Reserves minus Gold; ST Debt: Short-term External Debt; Priv Res: Net Private Reserves. 
with $\alpha=\beta=1$ which coincides with a uniform prior on $[0,1]$ b) an (improper) normal prior for $\psi_{i}^{N S S}$ and an (improper) inverse gamma prior for $\left(\sigma_{e, i}^{N S S}\right)^{2}$ that lead to posteriors that depend only on the data (see Kim and Nelson (1999)) c) a truncated (improper) normal and an inverse (improper) gamma prior for $\psi_{i}^{S S}-\psi_{i}^{N S S},\left(\sigma_{e, i}^{S S}\right)^{2}$ as explained in Kim and Nelson (1999). Finally, we assume that all priors are independent of each other. By well known results in Bayesian Statistics the posterior distributions are in the same class as these conjugate priors. Moreover there are simple closed form expressions for the parameters of the posterior distributions. This allows us to determine the posterior distribution of the parameters for each country separately. In the spirit of the Gibbs Sampler, when we update the posterior probabilities one at a time (i.e. keeping all parameters except one fixed). Finally, once we have determined the posterior distribution of each parameter, we make a random draw from that distribution and keep that number fixed until the next iteration of the sampler.

The updating of $p(N S S \rightarrow S S)$, and $p(S S \rightarrow N S S)$ is done by pooling the observations for all the countries. So, for each country we count the number of transitions to and out of sudden stops, and the total number of periods in normal times and in sudden stops. We then add all the episodes for all countries and find the posterior distributions as follows

$$
p(N S S \rightarrow S S) \sim \operatorname{beta}\left(1+\sum_{i} a_{i}^{S S}, 1+\sum_{i} a_{i}^{N S S}\right)
$$

where $a_{i}^{S S}$ is the number of observations marked as normal years that are followed by a year marked as sudden stop. We shall refer to this as a transition to a sudden stop. Conversely, $a_{i}^{N S S}$ counts the times that a normal year is followed by another normal year (NSS). This count is done country by country, but then we add all them up into a single number which is used in the updating process. Let us emphasize that this approach exploits the panel dimension of the data in order to obtain precise estimates of the transition probabilities.

Similarly, the posterior for the other parameter in the transition matrix is given by the following formula

$$
p(S S \rightarrow N S S) \sim \operatorname{beta}\left(1+\sum_{i} b_{i}^{N S S}, 1+\sum_{i} b_{i}^{S S}\right)
$$

where $b_{i}^{N S S}$ is the number of observations marked as sudden stops that are followed by a year marked as "normal". Conversely, $b_{i}^{S S}$ represents the other case, which is when a transition out of a sudden stop does not occur.

We conclude by making a random draw from these distributions too.

We record the random draws of a) the paths of 1's and 0's for each country, b) the country specific parameters $\left\{\psi_{i}^{N S S}, \psi_{i}^{S S}-\psi_{i}^{N S S}, \sigma_{e, i}^{N S S}, \sigma_{e, i}^{S S}\right\}$ and c) the "pooled estimates" of $p(N S S \rightarrow S S)$, and $p(S S \rightarrow$ $N S S$ ) . Then we repeat the above procedure several times and at each time we record the new draw of the paths of 1's and 0's for each country and the parameters. By properties of the Gibbs sampler, the 
posterior distribution of these random draws coincides in law with the posterior (joint) distribution of all the parameters (and the hidden states).

Section 5.1: In order to choose the cutoff point $x$ we proceeded as follows. First, we estimate an AR(1) process for $\log (V I X)$ and focus on the residuals $z_{t}$, which -according to the model- follow approximately a mixture of normals. Second we estimate a mixture of normals distribution for $z_{t}$. Having an estimate of the $p_{V I X}, \mu_{V I X}, \sigma_{V I X}^{2}, \mu_{\phi}, \sigma_{\phi}^{2}$, we then proceed to determine the cutoff in the same way that one would proceed in hypothesis testing. Namely, given our estimate of $\mu_{V I X}, \sigma_{V I X}^{2}$ we set the threshold $x$ high enough so that:

$$
P\left(z_{t}>x \mid J=0\right)<1 \%
$$

In statistical terms this would correspond to the "size" of a test that would (wrongly) accept the hypothesis of a jump with probability less than $1 \%$. Also, with the value of $x$ in hand, we can identify the months when the VIX actually crossed the threshold and we label them as jumps.

Let us reiterate that we adopt this procedure in order to be able to assess the performance of realistic hedging contracts. We also tried an alternative approach in order to determine $\lambda_{J}$ and $p_{J, S S}$ jointly: We ran a Gibbs Sampler to determine $p_{V I X}, \mu_{V I X}, \sigma_{V I X}^{2}, \mu_{\phi}, \sigma_{\phi}^{2}$ and the posterior probability of a jump in $z_{t}$. Subsequently we used the estimate of $p_{V I X}$ to determine $\lambda_{J}$. In a next step we sampled (jointly) from the posterior distribution of the states of $\widetilde{\psi}_{i}$ in section 3.1 and the posterior probabilities of a jump in the VIX to determine $p_{J, S S}$. Under either procedure we obtained similar results. We adopt the first procedure because it is the more conservative of the two and is closer to how such contracts would be implemented in practice.

Section 5.2: By using the cutoff value for the $V I X$ we also determine the months in which we observed a jump in the $V I X$. By that we mean months when the residuals of the estimated AR(1) process of the VIX exceeded the cutoff value $x$.

To determine a distribution of $p(S S \mid J)$ we proceed as follows. First, for each country we draw paths from the posterior distribution of the states $(N S S, S S)_{i}$, as provided by the Gibbs Sampler. Given the model, the only relevant observations for the conditional probability are the ones when the country $i$ is in NSS or has just transitioned to a $S S$. Hence, in accordance with the data generating process of the model, we discard all the quarters in which the country is in $S S$, except the one that marks the beginning of each $S S$. Then we look at all those times where the states switch from $N S S$ to $S S$ and simultaneously there is a jump in the VIX either in that quarter or the quarter before. We allow this short window to allow for the possibility of delayed data reporting etc. Let this number be given by $n_{S S, J}^{i}$. Similarly, we also determine all the times when there was a jump in the VIX. Let this number be $n_{J}^{i}$. Finally, define $n_{N S S+t}^{i}$ as the numbers of observations when country $i$ is either in $N S S$ or just moved to a $S S$ (a transition, $T$ ). We repeat this procedure for each country separately. 
After completing the above procedure for all countries we sum $n_{S S, J}^{i}, n_{J}^{i}$, and $n_{N S S \text { or } T}^{i}$ across countries. In accordance with the Bayesian methodology that we have been using throughout, we use a beta distribution with flat priors for both $p(J \mid N S S$ or $T)$ and $p(T \mid(N S S$ or $T)$ and $J)$. In exactly the same way that we updated the posteriors for $p(N S S \rightarrow S S)$, and $p(S S \rightarrow N S S)$ in section 3.1 we obtain the posterior distributions for the parameters $p(J \mid N S S$ or $T)$ and $p(J$ and $T \mid(N S S$ or $T)$ and $J)$, which are given by:

$$
\begin{aligned}
p(J \mid N S S \text { or } T) & \sim \operatorname{beta}\left(1+\Sigma_{i} n_{J}^{i}, 1+\Sigma_{i} n_{N S S \text { or } T}^{i}\right) \\
p(T \mid(N S S \text { or } T) \text { and } J) & \sim \operatorname{beta}\left(1+\Sigma_{i} n_{S S, J}^{i}, 1+\Sigma_{i} n_{J}^{i}\right)
\end{aligned}
$$

In a nutshell, for each iteration of the Gibbs sampler, we determine the number of times when a jump in the VIX coincided with a transition into a SS for each country. Then we pool across countries. This effectively imposes the constraint that all countries have the same joint probability distributions between transitions into a SS and jumps in the VIX. The benefit is that we can obtain more accurate estimates.

As a robustness check we also computed $p(J \mid N S S$ or $T)$ and $p(T \mid(N S S$ or $T)$ and $J)$ without imposing any prior, i.e. by just recording the random draws of:

$$
\begin{aligned}
p^{(k)}(J \mid N S S \text { or } T) & =\frac{\Sigma_{i} n_{J}^{i}}{\Sigma_{i} n_{N S S \text { or } T}^{i}} \\
p^{(k)}(T \mid(N S S \text { or } T) \text { and } J) & =\frac{\Sigma_{i} n_{S S, J}^{i}}{\Sigma_{i} n_{J}^{i}}
\end{aligned}
$$

at each iteration $k$ of the Gibbs Sampler and computing the empirical mean of the corresponding stationary distribution. The two approaches delivered very similar results, suggesting that our results are not influenced by the assumption of a uniform prior.

\section{Details on the numerical procedure}

To solve the model numerically, we proceeded as in Kushner and Dupuis (2001). The procedure is explained in great detail in Section 5.2 of that book and hence we only provide a sketch. The basic idea is to approximate the derivatives in all Bellman equations by discretizations. This way one can reexpress the value function at each point as an appropriately probability weighted average of the value function evaluated at neighboring points in the state space. That way the discretized version of the Bellman equation coincides with the solution to a particularly simple dynamic programming equation in discrete time, where the processes can only transit to neighboring states. For the exact formulas of the transition probabilities as well as the treatment of jumps we refer the reader to Kushner and Dupuis (2001) Ch. 13.2. Once this simple Markov chain has been determined, determination of the Value function can proceed by the standard value function iteration procedure. 


\section{Proofs}

Proof. (of Lemma 1) Post development the country's total available resources $A_{t}$ follow a geometric Brownian motion which up to a constant coincides with $Y_{t}$. Let us use the normalization:

$$
\widetilde{c}_{t}=\frac{C_{t}}{A_{t}}, \widetilde{x}_{t}=\frac{C_{t}}{A_{t}}
$$

where $A_{t}$ denotes post development resources. As we establish in section 2.3 the country's problem (in the developed phase) can be reduced to the solution of an essentially one dimensional problem with HJB equation

$$
\begin{aligned}
0= & \max _{\widetilde{c}_{t}}\left\{\frac{\widetilde{c}_{t}^{1-\gamma}}{1-\gamma}\right\}-\left(r-\mu_{Y}(1-\gamma)+\gamma(1-\gamma) \frac{1}{2} \sigma_{Y}^{2}\right) v^{G}+\left(\left(r-\mu_{Y}+\gamma \sigma_{Y}^{2}\right) \widetilde{x}_{t}+1-\widetilde{c}_{t}\right) v_{x}^{G} \\
& +\frac{1}{2} \sigma_{Y}^{2}\left(\widetilde{x}_{t}^{2} v_{x x}^{G}\right)
\end{aligned}
$$

This HJB is satisfied irrespective of the presence of any constraint. Assume now that, at $\widetilde{x}=0$ it is optimal to set

$$
\widetilde{c}_{t}^{*}=1
$$

i.e. the optimal level of consumption is set to be equal to income, since

$$
1=\widetilde{c}_{t}^{*}=\frac{C_{t}}{A_{t}}
$$

and accordingly:

$$
\frac{d \widetilde{x}_{t}}{d t}=0
$$

and similarly:

$$
\frac{d u^{\prime}\left(\widetilde{c}_{t}^{*}\right)}{d t}=0
$$

By equation (3.8) in Yong and Zhou (1999) it follows that the above equation can only be satisfied if:

$$
\left(r-\mu_{Y}(1-\gamma)+\gamma(1-\gamma) \frac{1}{2} \sigma_{Y}^{2}\right)=\left(r-\mu_{Y}+\gamma \sigma_{Y}^{2}\right)
$$

or:

$$
\mu_{Y}-\frac{\gamma+1}{2} \sigma_{Y}^{2}=0
$$

If the parameters satisfy this restriction, then the Euler equation will be satisfied with equality, and starting from $X_{t}=0$, the policy that sets $\widetilde{c}_{t}^{*}=1$ will be optimal.

For stronger growth or smaller volatility, $X_{t}=0$ will be binding as a constraint, starting from $X_{t}=0$. 


\section{References}

Aiyagari, S. R. (1994): "Uninsured Idiosyncratic Risk and Aggregate Saving," Quarterly Journal of Economics, 109(3), 659-684.

Arellano, C., and E. G. Mendoza (2002): "Credit Frictions and 'Sudden Stops' in Small Open Economies: An Equilibrium Business Cycle Framework for Emerging Markets Crises," NBER Working Papers: 8880 .

Broner, F., G. Lorenzoni, and S. Schmuckler (2003): "Why Do Emerging Economies Borrow Short Term?," mimeo. MIT Department of Economics.

Caballero, R. J., and A. Krishnamurthy (2001): "International and Domestic Collateral Constraints in a Model of Emerging Market Crises," Journal of Monetary Economics, 48(3), 513-548.

(2005): "Bubbles and Capital Flow Volatility: Causes and Risk Management," mimeo. MIT Department of Economics.

Caballero, R. J., and S. Panageas (2004): "Contingent Reserves Management: An Applied Framework," NBER Working Papers: 10786.

Calvo, G. A. (1998): "Capital Flows and Capital-Market Crises: The Simple Economics of Sudden Stops," Journal of Applied Economics, 1(1), 35-54.

Calvo, G. A., A. Izquierdo, and L.-F. Mejia (2004): "On the Empirics of Sudden Stops: The Relevance of Balance-Sheet Effects," NBER Working Papers: 10520.

Calvo, G. A., and C. M. Reinhart (1999): "Capital Flow Reversals, the Exchange Rate Debate, and Dollarization," Finance and Development, 36(3), 13-15.

Campbell, J. Y., and J. H. Cochrane (1999): "By Force of Habit: A Consumption-Based Explanation of Aggregate Stock Market Behavior," Journal of Political Economy, 107(2), 205-251.

Duffie, D., W. H. Fleming, H. M. Soner, and T. Zariphopulou (1997): "Hedging in Incomplete Markets with HARA Utility," Journal of Economic Dynamics and Control, 21(4-5), 753-782.

Duffie, J. D., and C. fu Huang (1985): "Implementing Arrow-Debreu Equilibria by Continuous Trading of Few Long-lived Securities," Econometrica, 53(6), 1337-1356.

El Karoui, N., And M. Jeanblanc-Picque (1998): "Optimization of Consumption with Labor Income," Finance and Stochastics, 2(4), 409-440.

Froot, K. A., D. S. Scharfstein, and J. C. Stein (1993): "Risk Management: Coordinating Corporate Investment and Financing Policies," Journal of Finance, 48(5), 1629-1658. 
Hamilton, J. D. (1989): "A New Approach to the Economic Analysis of Nonstationary Time Series and the Business Cycle," Econometrica, 57(2), 357-384.

- (1990): "Analysis of Time Series Subject to Changes in Regime," Journal of Econometrics, 45(1-2), 39-70.

He, H., and H. F. Pages (1993): "Labor Income, Borrowing Constraints, and Equilibrium Asset Prices," Economic Theory, 3(4), 663-696.

Heller, H. R. (1970): "Wealth and International Reserves," Review of Economics and Statistics, 52(2), 212-214.

Kim, C.-J., And C. R. Nelson (1999): State-space models with regime switching: Classical and Gibbssampling approaches with applications. MIT Press, Cambridge and London.

Kiyotaki, N., And J. Moore (1997): “Credit Cycles," Journal of Political Economy, 105(2), 211-248.

Kletzer, K., D. M. Newbery, and B. D. Wright (1992): "Smoothing Primary Exporters' Price Risks: Bonds, Futures, Options and Insurance," Oxford Economic Papers, 44(4), 641-671.

Kletzer, K. M., and B. D. Wright (2000): "Sovereign Debt as Intertemporal Barter," American Economic Review, 90(3), 621-639.

Kushner, H., And P. Dupuis (2001): Numerical Methods for Stochastic Control Problems in Continuous Time. Springer, New York.

LEE, J. (2004): "Insurance Value of International Reserves," mimeo. IMF.

Yong, J., And X. Y. Zhou (1999): Stochastic Controls: Hamiltonian Systems and HJB Equations. Springer, New York.

Zellner, A. (1997 [1988]): Bayesian Analysis in Econometricspp. 444-467, Statistical foundations for econometrics. Elgar; distributed by American International Distribution Corporation, Williston, Vt., Elgar Reference Collection. Foundations of Probability, Econometrics and Economic Games, vol. 5. Cheltenham, U.K. and Lyme, N.H. 